Щ्)FRANÇAISE

$>\mathrm{DE}$

필 PÉDAGOGIE

\section{Revue française de pédagogie}

Recherches en éducation

177 | octobre -décembre 2011

La politique ZEP en France, laboratoire des politiques

d'éducation?

\title{
Le déploiement des ENT dans l'enseignement secondaire : entre acteurs multiples, dénis et illusions
}

The implementation of digital workspaces in secondary education: between

various stakeholders, denials and illusions

El despliegue des ENT en la enseñanza secundaria; entre actores múltiples, denegaciones e ilusiones

Die Entwicklung des digitalen Arbeitsumfeld in der Sekundarstufe: zwischen zahlreichen Teilnehmer, Verleugnung und Illusionen

Éric Bruillard

\section{CpenEdition Journals}

Édition électronique

URL : http://journals.openedition.org/rfp/3410

DOI : $10.4000 /$ rfp.3410

ISSN : 2105-2913

Éditeur

ENS Éditions

Édition imprimée

Date de publication : 15 décembre 2011

Pagination : 101-130

ISBN : 978-2-84788-353-4

ISSN : 0556-7807

Référence électronique

Éric Bruillard, «Le déploiement des ENT dans l'enseignement secondaire : entre acteurs multiples, dénis et illusions », Revue française de pédagogie [En ligne], 177 | octobre -décembre 2011, mis en ligne le 11 décembre 2015, consulté le 10 décembre 2020. URL : http://journals.openedition.org/rfp/3410 ; DOI : https://doi.org/10.4000/rfp.3410 


\title{
NOTE DE SYNTHÈSE
}

\section{Le déploiement des ENT dans l'enseignement secondaire : entre acteurs multiples, dénis et illusions \\ Éric Bruillard}

\begin{abstract}
" Je voudrais seulement porter témoignage de la difficulté d'être qu'éprouve le décideur, le chercheur, ou l'utilisateur de la technologie éducative, laminé qu'il est entre les marchands et les prophètes dans un domaine où l'innovateur industriel vient proposer des solutions dont il reste encore à inventer le problème. » (Dieuzeide, 1982, p. 1)
\end{abstract}

Les espaces numériques de travail (ENT), déployés dans les établissements d'enseignement secondaire, peuvent être considérés comme une innovation institutionnelle. Ils constituent un cas d'étude intéressant pour les recherches consacrées aux technologies (informatiques) dans l'éducation, faisant intervenir toutes les caractéristiques de ce champ de recherche, notamment une imbrication très profonde entre des aspects politiques, économiques, techniques, sociaux et pédagogiques (ou didactiques). S'ajoutent des éléments de complexité, en raison de la multiplicité des acteurs concernés (ministère, académies, collectivités locales, Caisse des dépôts et consignations, entreprises de développement informatique, éditeurs scolaires, chefs d'établissement, enseignants...) dans des opérations de grande ampleur qualifiées d'industrielles. Les recherches menées sur les ENT révèlent les écarts importants entre les discours multiples de ces différents acteurs, l'état d'avancement du déploiement et les perceptions que peuvent en avoir des acteurs des établissements. Ces discours sont sous-tendus par des visions du déploiement technologique peu étayées cachant difficilement des formes de déni de certaines spécificités de l'éducation. Les recherches ont un rôle important à jouer pour dévoiler les tensions sous-jacentes à ces opérations et contribuer à ouvrir et nourrir les débats entre tous les acteurs.

Mots-clés (TESE) : recherche en éducation, TIC, utilisation de l'informatique, réseau informatique, objet pédagogique numérique, innovation pédagogique.

Les recherches consacrées aux technologies en éducation - concernant l'enseignement programmé, l'audiovisuel éducatif, l'enseignement assisté par ordinateur, Internet pour l'éducation, etc., pour citer quelques exemples de vagues d'innovations 
- se sont succédé depuis plus d'un demi-siècle. Si elles ont produit des résultats, on peut déplorer l'amnésie récurrente qui frappe la plupart des acteurs et des institutions s'agissant de l'« intégration » des toujours " nouvelles » technologies dans les systèmes éducatifs. Depuis assez longtemps (voir par exemple Cuban, 1986), il semble bien établi que l'informatisation dans les entreprises et dans les administrations n'est en rien comparable avec ce qui peut se produire dans les systèmes éducatifs pour ce qui est directement lié à l'enseignement et l'apprentissage. Pourtant, les « réussites » ou ce qui est considéré comme tel en entreprise seraient encore, pour les techno-réformateurs, des modèles pour le secteur éducatif. Peut-on discerner dans cette position l'impact du développement sans cesse croissant des industries éducatives (Mœglin, 2010), un énième avatar des marchands et des prophètes en technologie de l'éducation (Dieuzeide, 1982) ? Ou peut-être faut-il voir un autre processus à l'œuvre, conduisant à la " modernisation » de l'éducation, mais surtout du système scolaire lui-même, voire à la modification des modèles scolaires, comme le prédisent ou le préconisent différents textes états-uniens (Quentin, 2011) ? Pour expliquer l'amnésie des acteurs, le manque de cumulativité pourrait être reproché de même que le manque de diffusion des résultats et des méthodes des recherches dans le domaine des technologies en éducation. Ainsi, s'il est clair que l'association entre les "nouvelles » technologies et la motivation en éducation est éphémère et illusoire, puisqu'elle repose justement sur une nouveauté qui ne dure pas et une rareté qui disparaît dans la généralisation, elle est sans cesse revendiquée.

Toutefois, les travaux dont nous disposons ainsi que les discours des acteurs institutionnels pointent un résultat qui paraît à peu près admis, le constat de l'insuffisance du déterminisme technologique, le fait que l'utilisation sociale des technologies ne peut s'expliquer uniquement par les caractéristiques de la technologie elle-même. Mais ce résultat est repris, pour nombre de donneurs d'ordres, sous une forme légèrement amendée : comme la mise à disposition des configurations techniques n'est pas suffisante, quelles incitations et quels accompagnements faut-il mettre en œuvre afin que la " généralisation de la diffusion " s'opère ?

Dans ce texte, nous allons nous focaliser sur un « dispositif » particulier, les ENT (espaces numériques de travail), et faire le point sur les recherches qui leur sont consacrées. Leur étude semble caractéristique des recherches sur les technologies (informatiques) dans l'éducation. En effet, les ENT mobilisent de très nombreux acteurs de différents statuts, font l'objet de multiples discours et concentrent les éléments qui contribuent aux caractéristiques de ce champ de recherche : une imbrication très profonde entre des aspects politiques, économiques, techniques, sociaux et pédagogiques (ou didactiques). Toutefois, la manière dont ces ENT se déploient sur le territoire français conduit à repenser certains questionnements. D'abord, nous allons présenter le contexte français et les acteurs institutionnels qui financent et organisent l'éducation scolaire, puisque la répartition des compétences donne le cadre de déploiement des innovations technologiques dans l'éducation. Ensuite, quelques repères historiques nous permettront de cerner la notion d'ENT et son évolution ainsi que les implications et les contraintes qui en découlent pour la recherche. Puis nous présenterons les recherches qui ont été menées. Nous discuterons ensuite des cadres théoriques et méthodologiques utilisés par quelques chercheurs, mais qui correspondent aux idées dominantes des acteurs institutionnels en essayant de révéler leurs présupposés. La réflexion porte ici essentiellement sur l'enseignement du second degré, les questions se posant de manière différente à l'université et plus largement dans l'enseignement supérieur, notamment relativement à plusieurs dimensions : le rôle des parents, le statut des élèves, les questions de «pédagogie ", les problèmes de gouvernance et d'organisation, les modes de 
financement, etc. De même, nous nous centrons sur le cas français ; des comparaisons internationales, pour qu'elles soient intéressantes, demanderaient de longs développements, compte tenu du poids du contexte institutionnel.

\section{LE PILOTAGE DU SYSTÈME ÉDUCATIF ENTRE CENTRALISATION ET DÉCENTRALISATION}

On ne peut comprendre les ENT en éducation, du fait qu'ils dépendent de prescriptions institutionnelles mises en œuvre par des acteurs d'institutions différentes, sans brosser rapidement le cadre général de l'enseignement scolaire français. II faut d'abord souligner la prééminence de l'État et du ministère de l'Éducation nationale, s'agissant des personnels enseignants, des programmes, des examens, de la collation des grades. Mais, depuis la loi de décentralisation, les collectivités sont en charge des bâtiments, des équipements et de leur maintenance, de l'entretien des locaux, des personnels TOS (Techniciens et ouvriers spécialisés)... et des infrastructures réseaux. Comme le précise Alain-Marie Bassy ${ }^{1}$, la compétence éducative (c'est-à-dire la responsabilité confiée à une institution et assumée par elle) est souvent mal définie par les textes ou assumée au titre des compétences générales. Dans un cadre réglementaire peu explicite (sur les équipements informatiques ou sur les ressources numériques), une grande disparité des responsabilités assumées par les collectivités peut être observée. Compliquant encore le tableau, trois niveaux de collectivités (commune, département, région) ont chacun en charge un niveau de l'enseignement scolaire (école, collège, lycée). Un élève passant d'une école à un collège puis à un lycée dans le même territoire dépend successivement des différents niveaux de ces collectivités. Des politiques menées à chacun de ces niveaux, selon différents clivages politiques, conduisent souvent à un manque de cohérence des choix stratégiques et techniques et à un défaut d'articulation dans un parcours scolaire.

Sur la question des réseaux informatiques pour l'éducation, la majeure partie des prescriptions relève de la compétence de l'État alors que les questions de financement et de mise en œuvre relèvent des collectivités. L'État a le monopole des questions pédagogiques au sein des établissements, mais l'apparition des services numériques rend en grande partie caduque la distinction entre ce qui est pédagogique et ce qui ne le serait pas. Entre l'État et les collectivités, un troisième acteur joue un rôle central, la Caisse des dépôts et consignations (CDC). La CDC se présente comme « un groupe public, investisseur de long terme au service de l'intérêt général et du développement économique du pays ${ }^{2}$ ». Créée en 1816, placée sous le contrôle direct du Parlement, la CDC est une banque publique, exerçant des missions d'intérêt général pour le compte de l'État et des collectivités territoriales, assurant un lien entre eux, agissant parfois comme un tiers de confiance. La CDC intervient notamment sur la modernisation économique du pays et sur le développement numérique des territoires ${ }^{3}$, proposant aux collectivités territoriales une expertise qualifiée de «neutre 4 » et un appui financier. Elle a travaillé dès 1998 sur les espaces publics numériques, avec le développement du projet «Cyberbase ${ }^{5}$ ». Elle a eu logiquement une mission sur le haut débit au début des années 2000. II semble que pour mieux justifier la mise en place de cette infrastructure auprès des élus des collectivités, la CDC se soit intéressée aux services et aux usages facilités par le haut débit, ce qui l'a conduite ainsi à travailler au développement des espaces numériques de travail pour la communauté éducative. 
La répartition complexe des prérogatives en matière d'éducation que nous venons d'esquisser est source potentielle de difficultés et de conflits. Les réseaux informatiques et les ENT sont au cœur de tensions entre les différents acteurs institutionnels. D'autres particularités du système éducatif français jouent également un rôle important. Deux exemples semblent particulièrement caractéristiques. Le premier concerne les ressources éducatives et plus exactement les manuels scolaires. Si l'État définit les programmes, le système français est caractérisé par une triple liberté (Choppin, 2005) : liberté de la production, liberté du choix (par les enseignants), liberté de I'utilisation (liberté pédagogique des enseignants). Toute modernisation qui s'accompagne d'une certaine normalisation entre en conflit avec ces différentes libertés. Cela peut conduire à la contestation, par les enseignants, de choix techniques considérés comme contraignant leurs choix pédagogiques. Le second concerne les fichiers nominatifs. Comme le rappelle la $\mathrm{CNIL}^{6}$, " l'informatique doit respecter l'identité humaine, les droits de l'homme, la vie privée et les libertés ». Développer les réseaux informatiques dans l'éducation conduit à multiplier les traces d'activité des élèves et des enseignants et surtout facilite leur récupération. Or, l'un des intérêts des ENT est justement de fournir des données et de faciliter leur traitement. Qui peut constituer ces fichiers de données ? Qui peut les consulter ? Qui peut les traiter ? Un arrêté promulgué en 2006 sur les données personnelles et les ENT précise la liste de ces données et les contraintes de gestion?.

Des recherches seraient à conduire sur les politiques publiques en matière d'éducation et de TIC (Technologies de l'information et de la communication) pour aider à dénouer les interactions complexes entre les différents acteurs institutionnels et à situer les impacts de ces interactions sur le fonctionnement des établissements scolaires. L'une des questions, dans la compréhension de phénomènes locaux, au sein d'une classe ou d'un établissement, est de préciser de quelle manière ils peuvent être déterminés par d'autres échelons, de situer l'articulation entre des pratiques individuelles ou locales et des contraintes ou des cadres beaucoup plus larges.

\section{QU'EST-CE QU'UN ENVIRONNEMENT NUMÉRIQUE DE TRAVAIL ? POUR QUELS ACTEURS ?}

\section{Un ENT : point d'accès ou ensemble intégré de services ?}

L'ENT peut être considéré comme un portail de services offerts à la communauté éducative (enseignants, élèves, personnels administratifs, techniciens, mais aussi parents, intervenants extérieurs, etc.), accessibles à partir d'une identification unique (caractéristique importante des ENT), que ce soit à l'intérieur ou à l'extérieur de l'établissement : le bureau numérique (annuaire, espace de stockage, agenda, outils bureautiques) ; les outils de communication (courriel, accès Internet) ; les services de vie scolaire (emploi du temps, notes, absences, information administrative) ; les services pédagogiques et documentaires (ressources pédagogiques, dictionnaires et bases de données, outils de création, de publication et de collaboration).

Si différentes autres définitions assez similaires peuvent être consultées (par exemple, celle de la $C^{2} C^{8}$ ou celle du ministère en $2009^{9}$ ), une ambiguïté apparaît à leur lecture. L'ENT n'est-il qu'un point d'accès, un simple portail ou le système global, incluant un ensemble de services ? La courte histoire des ENT apporte quelques éléments de réponse. Cette histoire est toutefois un peu difficile à recons- 
tituer, les sources étant éparpillées et peu accessibles. En particulier, le site du ministère Educnet a été refondu avec le site Eduscol en septembre 2011 et de nombreuses pages ont été laissées de côté. II n'y a pas d'archivage systématique et on constate beaucoup de liens morts à partir des publications accessibles. Ces difficultés participent sans doute à l'amnésie des acteurs que nous avons pointée en introduction. Ainsi, le site actuel du ministère est peu prolixe. Dans une page consacrée à I'histoire des ENT ${ }^{10}$, sans lien Internet activable, les intertitres insistent sur l'importance de l'opération dans une progression constante vers la couverture complète du territoire national : «Des premiers déploiements massifs d'ENT... », « ... au déploiement à grande échelle... », “... vers la généralisation à l'ensemble des établissements scolaires ". Un long fleuve tranquille, somme toute! Toutefois, des généralisations ont plusieurs fois été annoncées par les autorités ministérielles (dès 2003 pour $2007^{11}$, en 2008 pour 201012, réaffirmées en 2010), avec un horizon qui a eu la fâcheuse tendance de s'éloigner sans cesse.

Gérard Puimatto (2006), dans sa thèse, fournit les repères initiaux quant à l'émergence des ENT dans la suite des réseaux pour l'éducation. À la fin des années quatre-vingt-dix, sous le vocable « bureau virtuel » (Gros, 2001), de nombreux travaux ont été diligentés pour procurer aux personnels de l'Éducation nationale un environnement de travail sur Internet personnalisé et personnalisable. D'autres travaux se sont orientés vers les élèves et les étudiants, sous le vocable "Cartable électronique ", notamment le Cartable de l'université de Savoie (Christian Martel), celui développé par Bordas et Nathan, l'i-manuel de la société Editronics, etc. Des premiers tests ont été effectués dans des établissements scolaires (Ginioux \& Narcy, 2002 ; Toulouse, 2002). Dans un rapport de 2002 (Kaplan, 2002), un groupe de travail réuni par la FING ${ }^{13}$ recensait douze projets faisant intervenir le concept de "Cartable électronique ", sous des formes très diverses, voire apparemment contradictoires, mais avec un point commun, "celui de faire passer l'usage de l'ordinateur à l'école de l'anonyme au personnel ". II s'agit de doter chaque enseignant et chaque élève d'un espace numérique personnel, enrichi d'outils et de contenus, et relié de différentes manières aux espaces collectifs que sont la classe, l'établissement, éventuellement la famille, ainsi que d'offrir un objet personnel nomade et d'organiser l'accès à des ressources distantes. À l'automne 2002, le ministre de l'Éducation demande à la Caisse des dépôts de mener des benchmarks sur les ENT - c'est-à-dire une étude comparative de plusieurs expériences étrangères - afin de faire émerger quelques grandes solutions industrielles. L'étude alors réalisée montre une grande hétérogénéité des solutions existantes, ce qui est considéré comme un frein à la mise en œuvre d'une cohérence nationale, d'autant plus que les initiatives locales paraissent généralement peu matures et, bien que fonctionnelles, sont souvent qualifiées de «bricolage » (Missonier, 2008, p. 196).

Un tournant correspond à l'appel à projets ENT lancé par la CDC et le ministère de l'Éducation en mars 2003, afin de mettre en place des projets communs de collectivités territoriales et de services déconcentrés de l'Éducation nationale (les rectorats). Huit projets ont été sélectionnés (Puimatto, 2004). Cela a conduit à un processus de conventionnement entre l'État, la CDC et les collectivités territoriales. Un dispositif de suivi et d'échange des projets ENT est également mis en place (dès septembre 2003) et un schéma directeur (le SDET ${ }^{14}$ ) est publié, précisant ce que doit être un ENT. Si ce schéma est le fruit d'une négociation entre différentes directions du ministère, plaçant des "impératifs d'usage en contrepoids aux impératifs d'infrastructure et de sécurité » (Puimatto, 2006, p. 395), il contribue à donner des pistes de régulation pour le pilotage des projets et à structurer la relation entre l'État et les collectivités. 
À partir de cette impulsion nationale, il est ensuite question d'expérimentation et surtout de déploiement (Pouts-Lajus, 2005). Les ENT “ officiels ", c'est-à-dire ceux qui respectent le schéma directeur SDET et sont agréés par le ministère, vont se focaliser sur les questions de vie scolaire - absences, notes, emplois du temps, réservation des salles et des équipements... Vont coexister des ENT officiels ou labellisés et d'autres non comptabilisés dans les statistiques. Cependant, en comparant les dernières cartes de déploiement des ENT proposées par la CDC et le ministère de l'Éducation, des divergences intéressantes émergent. La CDC fournit une carte interactive des marchés de généralisation des ENT ${ }^{15}$ alors que le ministère ${ }^{16}$ donne, pour chaque académie, le niveau d'avancement du ou des projets (projet en cours ou étude préalable, expérimentation, généralisation), ainsi que les partenariats éventuels avec les collectivités territoriales concernées. En particulier, certains projets académiques sélectionnés en 2003 n'apparaissent pas dans la carte de la CDC (Bordeaux, Poitiers, Dijon) mais sont présents dans la carte du ministère 17 : Poitiers et Dijon sont déclarés en expérimentation alors qu'Argos est présenté comme déployé à plus de $50 \%$ mais avec « une évolution en cours vers une nouvelle solution en partenariat avec le conseil régional ». La plupart des solutions retenues en 2003 ne sont pas celles déployées en 2012. Ainsi, les précurseurs de l'Éducation nationale en 2003 (portés par les académies) ont pour la plupart disparu et des marchés de généralisation ne sont pas encore en place. Peut-on y voir un signe de résistance des académies à la politique du ministère, des désaccords avec les collectivités territoriales?

Si une généralisation des ENT était annoncée pour 2007, il semble qu'un transfert possible des compétences de l'État vers les collectivités (avec les budgets et les personnes, notamment les informaticiens travaillant dans les rectorats) n'ait pas été opéré, conduisant à une situation complexe et instable. Par ailleurs, suite à l'initiative menée dans les Landes dès $2001^{18}$, des opérations de dotations d'ordinateurs portables pour les élèves vont être conduites dans différents départements (Khaneboubi, 2009 ; Rinaudo \& Poyet, 2009). En conséquence, notamment pour des raisons budgétaires, ces départements ne vont pas développer d'ENT.

Pour résumer, on est parti de premières "solutions " à des problèmes rencontrés par les acteurs de l'éducation, enseignants et élèves : le " bureau virtuel ", afin de répondre à certains aspects nomades du travail de l'enseignant (préparation à la maison, intervention dans des salles différentes et absence de bureau personnel au sein des établissements scolaires) ; le cartable (numérique, virtuel, en ligne...) pour les élèves, correspondant aux outils et ressources qu'ils doivent transporter de la maison à l'école, avec en arrière-plan la sempiternelle complainte du poids excessif du cartable. On en arrive à concevoir des espaces qui impliquent la mise en place de systèmes d'information au sein d'institutions. De point d'entrée, l'ENT est devenu le système d'information lui-même, censé offrir des " bouquets de services numériques » (MEN, 2010) et même un moyen de choisir et de structurer cette offre. L'ENT apparaît dès lors comme un moyen pour moderniser le fonctionnement de l'établissement scolaire ${ }^{19}$. Une vision tournée vers les acteurs eux-mêmes, au plus proche de leurs pratiques, se transforme en un schéma purement descendant, conduisant à s'interroger sur la manière de transformer les acteurs éducatifs en alliés d'une réforme institutionnelle. Aux interrogations pédagogiques se sont substitués des schémas gestionnaires (Puimatto, 2008).

\section{Les ENT, une innovation institutionnelle associant de nombreux acteurs}

Les ENT, ni innovation pédagogique ni innovation technique, constituent une innovation institutionnelle, dans lequel le processus même est central (Cros, 1997), visant 
un changement de modèle avec l'idée, qui présidait déjà aux beaux jours de l'enseignement programmé dans les années soixante, de passer de l'artisanat à l'industrie. Mais pour cela, une généralisation (de l'outil à une population scolaire d'un territoire, et de l'usage comme l'environnement de travail numérique quotidien) s'avère une condition nécessaire (Puimatto, 2008). Cette " généralisation » est conduite par un grand nombre d'acteurs et se situe au carrefour d'interrogations et de politiques multiples. Le ministère pilote indirectement les projets, par le biais du cadre de référence national des ENT qu'il construit et maintient (notamment via le schéma directeur des espaces numériques de travail), conduisant à des recommandations "déclinées en exigences par le biais des marchés publics passés par les partenaires locaux ". II organise " la mutualisation des expériences et des réflexions des différents porteurs de projet, afin d'accompagner les changements liés aux projets ENT » (MEN, 2010, p. 13). Récemment, le ministère a pris d'autres rôles : d'abord celui de certificateur20 (puisque ce sont les collectivités qui passent les marchés), puis celui de pilote d'un dispositif d'évaluation, non pas technique, mais des " usages ${ }^{21}$ ». Quant à la Caisse des dépôts, elle anime une cellule de travail nationale sur les ENT. Elle a également œuvré à des rapprochements entre les espaces publics numériques et l'école ${ }^{22}$, notamment dans le projet "Cyberbase " avec des implantations dans les écoles primaires, et elle est engagée dans d'autres chantiers (infrastructures haut débit, développement durable, universités numériques, etc.) conduisant à associer la question des ENT à d'autres développements. Ainsi, une étude menée en 2010, commanditée par la CDC, anticipant les débits pour l'utilisation d'Internet et des ENT dans les collèges et les lycées, conclut à la nécessité d'avoir recours à la fibre optique ${ }^{23}$.

Les collectivités sont sans doute désormais l'acteur essentiel, puisqu'elles ont la charge de l'achat et de la maintenance des matériels et des bâtiments, etc., et maintenant de celui des ENT. Ces derniers peuvent s'inscrire au sein de politiques régionales ou départementales de développement des technologies de l'information et de la communication. Ainsi, selon Charlotte Ullmann (2007), parlant du « millefeuille institutionnel français ", les régions, soucieuses de la compétitivité de leur territoire, peuvent envisager l'intégration des TIC « comme une opportunité, non seulement de renforcer leur positionnement parmi les autres collectivités, mais aussi comme un moyen de créer des stratégies efficaces en termes de développement local » (Ullmann, 2007, p. 113). Les liens entre les questions d'aménagement du territoire et le déploiement des TIC dans les établissements scolaires seraient certainement à étudier plus profondément.

En gros, les infrastructures relèvent de la collectivité et les usages, de l'établissement scolaire, sous la tutelle de l'État via les académies. Si l'organisation relève à la fois des établissements, de l'État et de la collectivité, demeure une forte prédominance de l'État en matière scolaire, notamment du fait que les collectivités n'ont aucune autorité dans ce qui relève de la pédagogie et des programmes. "Découlent de cette situation des jeux d'acteurs complexes, avec comme enjeu des questions de leadership et de cohérence institutionnelle " (Puimatto, 2008, p. 37), ce qui entraîne certaines difficultés dans les relations entre l'État et les collectivités (voir par exemple Chaptal, 2005).

\section{QUELLES CONTRAINTES SUR LES RECHERCHES ?}

\section{Des recherches qui ne peuvent pas être conclusives}

Commençons par une affirmation qui peut paraître très décevante. Les recherches ne peuvent pas statuer sur le bien-fondé des ENT en éducation. S'agissant d'un 
changement de système, il n'est pas possible de prévoir l'ensemble des modifications entraînées par ce changement et associées à lui. En particulier, augurer de changements sociaux est une tâche plus que périlleuse et ce qui peut accompagner ce processus n'est pas complètement déterminé. Tout au plus les recherches ontelles pu montrer des possibles, que telle ou telle fonctionnalité, tel ou tel instrument dans une certaine situation éducative avec certains acteurs se sont avérés bénéfiques pour certaines dimensions liées à l'apprentissage. On peut associer des résultats empiriques à des théories et des modèles montrant les apports possibles pour le système éducatif ou les situations de formation. Les méthodes expérimentales permettent de faire des choix locaux (tel type de présentation, telle modalité d'association texte-image, telle forme de redondance, etc.). Les grandes enquêtes fournissent des écarts et des pistes d'interprétation. En effet, beaucoup de recherches fonctionnent par différences, par comparaison de situations, à la condition que ces situations soient suffisamment voisines et que des variables extérieures n'aient pas un poids trop fort. Les recherches-actions, le design experiment, permettent d'étudier et d'affiner sur des temps longs (plusieurs mois ou plusieurs années), de confronter et de nourrir des approches théoriques vis-à-vis de pratiques. Mais la recherche en éducation ne peut statuer sur ce qui n'existe pas encore ou dans un état par trop embryonnaire.

Un tel résultat - neutre, voire négatif - ne permet pas de légitimer les choix technologiques et organisationnels qui ont été faits. Ce que peut faire la recherche, c'est documenter, rendre intelligibles les processus de transformations en cours. Ce qui peut être bien évidemment créateur et permet d'aider à choisir des orientations. Mais les acteurs des ENT (ministère, CDC, collectivités, sociétés informatiques) sont-ils prêts à tenir compte de ces informations et le peuvent-ils ? Leurs systèmes de contraintes leur laissent-ils suffisamment de choix ?

\section{Une recherche d'accompagnement marginalisée, à la recherche des " bons usages"}

Le point le plus évident, s'agissant des ENT, est que la recherche n'apparaît pas en amont en tant que telle. Pour les orientations générales, ce sont des experts, des groupes de réflexion ou de pression qui « piochent » dans la littérature de recherche pour produire des discours. Ne se diffusent que quelques données chiffrées abondamment reprises et des exemples d'initiatives alimentant la rhétorique d'un prétendu retard français, invitant à des politiques massives d'équipement (Bruillard, 2011).

Pourtant, s'agissant de mieux comprendre ce qui se fait dans d'autres pays, des études ciblées sont menées produisant des résultats fructueux pour nourrir les réflexions. S'agissant des ENT, bien que les comparaisons soient difficiles, puisque des correspondances sont nécessairement à faire (la notion même d'ENT est spécifique à la France), plusieurs études ont été réalisées. D'abord, celle que l'on pourrait qualifier d'étude princeps, conduite par I'IDATE (Institut de l'audiovisuel et des télécommunications en Europe) et publiée en 2003, dont la publication a attesté une grande diversité de solutions. Les éléments ont été repris, notamment dans divers rapports et articles (Kaplan \& Pouts-Lajus, 2004a). Les travaux d'Alain Chaptal centrés sur les États-Unis et le Royaume-Uni donnent des analyses détaillées sur les politiques menées dans ces deux pays (Chaptal, 2007a, 2008). Des études récentes et ciblées, commandées par la CDC, décrivent le marché international de la e-éducation (CDC \& Cap Digital, 2010) et font le point sur les plates-formes virtuelles d'apprentissage en Europe (CDC, ministère de l'Éducation nationale \& European Schoolnet, 2010). Une recommandation de cette dernière étude devrait guider les 
processus de déploiement en France : "Trois facteurs clés de succès se dégagent : la participation des enseignants à l'ensemble du processus depuis sa conception et tout au long de sa mise en œuvre, le pragmatisme et la patience au service d'un déploiement qui a besoin de temps, et enfin la mise à disposition des moyens nécessaires (support technique, formation, communication, financement, etc. ${ }^{24}$ ). " Mais, comme nous le verrons, les recherches menées montrent justement que ces facteurs ne semblent pas être pris en compte dans les processus de déploiement.

Les appels d'offres (pour les marchés de généralisation) sont rédigés et les «meilleurs répondants " sont sélectionnés, à partir de cahiers des charges complexes. La recherche arrive éventuellement après, elle doit trouver sa place au sein d'un processus déjà engagé, l'invitant à tenir au mieux un rôle d'accompagnement. Le problème est alors plutôt de l'ordre du management, de la conduite du changement dans les institutions. Les modèles utilisés vont venir de l'entreprise et on va retrouver le schéma classique d'application à l'éducation des méthodes issues d'autres secteurs. Bon usage et bonnes pratiques sont les maîtres-mots des institutions qui gèrent le déploiement des ENT.

Toutefois, les acteurs institutionnels, notamment les collectivités qui assurent le financement, ont besoin d'une sorte de mesure de leur action. Ce n'est pas sans poser problème. En effet, la nature des ENT et leur définition fluctuante conditionnent la manière de juger de leur impact. Comment juger de l'amélioration de la relation avec les parents d'élèves ? Comment juger de l'amélioration de la vie scolaire ? Par exemple, si on constate une baisse de l'absentéisme, pourrait-on l'attribuer à l'ENT ? Si ce dernier peut permettre des signalements plus rapides, de rendre compte plus facilement aux autorités de tutelle, il n'est qu'un des maillons rendus indispensables dans des formes de gestion à base d'indicateurs. Une réduction des coûts pourrait être mise en évidence ${ }^{25}$, mais il faudrait une comptabilité fine (décharges, temps de formation, etc.) pour consolider les évaluations financières.

En tant qu'intégrateurs de services, comment évaluer leur apport autrement qu'en étudiant la progression de leur utilisation ? L'indicateur majeur serait le nombre d'utilisateurs, ou plus finement, le nombre de connexions. La CDC a justement mis en place un dispositif de marquage des différentes plateformes d'ENT avec une correspondance entre les services offerts par les différentes solutions ENT (Coutellier, Richard, Le Loup et al., 2010). Grâce à ce dispositif, elle peut procurer aux collectivités des tableaux de bord statistiques afin de suivre la progression des statistiques d'utilisation et leur permettre d'orienter leur stratégie de déploiement. Cette initiative est importante, d'une part parce qu'elle permet d'obtenir des données d'utilisation indépendantes des fournisseurs, d'autre part parce qu'elle incite à des comparaisons sur le plan territorial, voire national.

Ce type de mesure d'audience ou de participation, mis en place par la Caisse des dépôts, répond bien aux contraintes des ENT, compte tenu de la répartition des pouvoirs entre les différentes autorités. Toutefois, ces mesures ont un inconvénient, si elles sont les seules utilisées pour juger de la "réussite " des ENT. En effet, on risque de confondre utilisation et pertinence. Si les personnes ciblées utilisent effectivement les services proposés et si les taux sont en progression, on a fait la "preuve " de l'intérêt de l'innovation ; aux acteurs ensuite de trouver les "bonnes utilisations ". Chacun reste dans son rôle et dans son domaine : on chiffre des visites. Mais l'augmentation des taux d'utilisation peut cacher des usages finalement peu intéressants. Un deuxième problème est le fait que ces données ne sont pas véritablement publiques et pas forcément disponibles pour la recherche.

Un appel à projets a été lancé par la CDC afin de compléter les mesures d'audience par des recherches plus qualitatives, avec une demande de cofinancement 
des collectivités à l'automne 201026. Mais les collectivités vont-elles s'intéresser à ces recherches, surtout si les solutions déployées ne fonctionnent pas aussi bien ou ne s'avèrent pas aussi pertinentes que souhaité ? Les données de mesure d'audience seront-elles rendues publiques si elles confirment une adoption tardant trop à venir? Notons d'ailleurs qu'il n'y a pas encore de publication utilisant les données quantitatives fournies par les dispositifs de marquage mis en place à l'initiative de la CDC, à l'exception d'un premier travail sur l'académie de Nancy-Metz (Schneewele, Cherqui-Houot, Nowakowski et al., 2010).

\section{LES RECHERCHES SUR LES ENT : DES PROBLÈMES BIEN CERNÉS VIA DIFFÉRENTES APPROCHES}

Nous allons brièvement présenter une cartographie des recherches qui se sont déroulées ou qui sont en cours. Suite aux deux journées organisées à l'ENS Cachan ${ }^{27}$ ayant pour but de faire le point sur les recherches menées sur les ENT dans l'enseignement secondaire et de contribuer à faire émerger un milieu de recherche sur ces questions, un premier recensement des recherches a pu être mené. II a été complété par un relevé systématique effectué à partir des références citées dans les travaux présentés. Les recherches ne sont pas si nombreuses, plutôt éparpillées, mais leur mise en perspective fournit des analyses très précieuses sur les ENT et des résultats très convergents. Les travaux portent autant sur les impacts ou les activités mises en œuvre en classe et dans les établissements scolaires que sur le suivi des opérations de déploiement et l'étude des discours et des actions des différents acteurs impliqués. Peu de thèses : le travail pionnier de Gérard Puimatto (2006), dont les ENT ne constituent qu'une partie, et celui de Delphine Billouard (2011), mais qui concerne les ENT dans les universités, enfin les thèses de Stéphanie Missonier (2008) et Emmanuelle Voulgre (2011). Les deux premières sont inscrites en sciences de l'information et de la communication et s'attachent à décrire les projets de déploiement selon plusieurs dimensions, notamment du côté de leur pilotage. La thèse de Stéphanie Missonier, en sciences de gestion, analyse les stratégies mises en œuvre par les décideurs et les concepteurs (les entreprises) dans le déroulement de l'implantation d'un ENT.

Emmanuelle Voulgre, en sciences de l'éducation, propose un panorama très large des TIC pour l'éducation dans le système scolaire, avec une approche systémique. Sur la base de revues de la littérature de recherche et d'entretiens avec des acteurs (enseignants, élèves, membres des équipes de direction), elle interroge différents services offerts par l'ENT et dévoile certaines tensions : est-il un espace documentaire au service de la pédagogie ? Le cahier de textes en ligne peut-il devenir un outil pour la personnalisation des travaux des élèves ? Quel impact peut avoir l'utilisation d'un ENT sur les conseils de classe, le contrôle de l'assiduité des élèves, le soutien scolaire ? Si les travaux ne sont pas conclusifs, ils donnent la parole aux acteurs et montrent que beaucoup de questions n'ont pas été anticipées avant la mise en place de l'ENT. L'auteur conclut (Voulgre, 2011, p. 258) sur le constat de l'absence systématique de projet pédagogique d'établissements associé à chacun des modules ou reliant plusieurs modules de I'ENT. 


\section{Les études autour du Cartable de Savoie : un tour d'horizon des problématiques}

Le Cartable électronique de Savoie, précurseur en 1999, a donné lieu à différents travaux. D'abord, une lignée de recherches plutôt informatiques sur la conception du Cartable, puis sur les activités collaboratives et les scénarios pédagogiques (Martel \& Vignollet, 2001 ; Martel, Ferraris, Caron et al., 2004 ; Caron, Carron, Chabert et al., 2004, etc.). De premiers rapports sur les utilisations (Toulouse, 2002) ont suivi, puis une analyse systémique de l'opération "Cartable numérique » de Grenoble. Un travail similaire avait été réalisé par Anne Romby (2001) à la fin des années quatrevingt-dix, sur l'analyse des dynamiques en jeu, des stratégies des acteurs concernés par le projet «Éducapôle » en Picardie. Dans leur analyse systémique, Collet (2004) et Collet, Anselm, Narvor et alii (2007) décrivent le processus de mise en place de l'innovation, les points d'achoppement (les " controverses ") entre différentes catégories d'acteurs et la prédominance des acteurs disposant du financement : un projet qui échoue, des changements de plateformes logicielles, des acteurs en déficit de confiance, etc. Ils soulignent le caractère difficilement conciliable des objectifs des différents acteurs, notamment la volonté des initiateurs, qui sont porteurs de conceptions pédagogiques novatrices, de maintenir les technologies à "l'écart des forces commerciales susceptibles de les dévoyer "(Collet, 2004, p. 10). Ainsi, s'agissant par exemple des ressources éducatives qui pourraient être associées, les personnes préconisant leur gratuité se sont heurtées à d'autres intérêts. L'analyse de Gérard Collet souligne l'existence de réticences " de fond " des acteurs finaux (les enseignants) face à ce qui leur apparaît comme une "incapacité majeure du système à monter, à soutenir et à piloter l'innovation attendue " (Collet, Anselm, Narvor et al., 2007, p. 10).

Le travail de Stéphanie Missonier permet de comprendre ce même processus d'innovation en l'étudiant du côté des entreprises. Se basant sur la théorie de l'acteur réseau (Akrich, Callon \& Latour, 2006), elle analyse notamment la formation et la dynamique des controverses au sein du réseau sociotechnique intervenant dans le projet de développement d'un ENT. L'échec de ce projet particulier est, selon elle, conséquence du manque de convergence induit par un enchaînement de controverses non stabilisées. Partant du constat initial de la difficulté, pour les entreprises, de travailler dans l'éducation, elle conclut à des recommandations concernant la conduite stratégique selon trois axes de coordination : le renforcement du réseau, la fiabilité et l'évolutivité de la technologie, ainsi que l'accompagnement des utilisateurs.

Toujours sur le Cartable numérique de Savoie, le projet «OUVRE » (Observation des usages des environnements et des ressources numériques pour l'éducation) conduit par Françoise Poyet et Sylvain Genevois ${ }^{28}$ interroge la tension entre un environnement informatique tourné vers les utilisateurs nomades (ressources accessibles hors de la classe) et l'environnement scolaire avec une organisation très fortement tributaire des contraintes de temps, de lieu et d'espace. Afin de rapprocher des cultures et des logiques très éloignées, celles des développeurs des ENT et celles des enseignants, ils montrent que le recours à des métaphores pour désigner des outils et des services informatisés mis à disposition des enseignants favorise en partie l'appropriation de ces outils. Les métaphores réduisent le décalage et permettent de mieux faire correspondre les contextes technologiques avec les contextes pédagogiques des enseignants. 
OUVRE s'est prolongé dans un projet intitulé «APPARENT » (Analyse des pratiques des professeurs et apprenants et des représentations dans les environnements numériques de travail29), avec un terrain élargi à deux académies et une prise en compte de l'ensemble du secondaire (collèges et lycées). L'analyse des huit cent cinquante réponses obtenues à un questionnaire structuré à partir des fonctionnalités offertes par les ENT ne vise pas, selon les auteurs (Genevois \& Poyet, 2009b), à mesurer l'état du déploiement des ENT ou à donner des indicateurs pour améliorer le pilotage des projets, mais s'intègre dans un projet plus vaste de recensement des usages que les enseignants font des ENT, d'analyse des facteurs influençant l'intégration des ENT, de développement de leurs usages et d'élaboration d'une typologie d'usagers. II ressort que les enseignants utilisent les fonctionnalités proposées de manière dissociée (pas de regroupement de plusieurs fonctionnalités pour une même tâche), « jouant » de l'ENT plutôt comme d'un portail de services, sans encore développer d'approche globale 30 .

La prise en compte du point de vue des acteurs finaux, ceux qui sont censés utiliser les ENT, via des entretiens, des questionnaires, voire des observations quand il s'agit d'utilisations en classe caractérise les recherches sur les ENT. Un premier résultat, dès 2003, concerne un Cartable numérique déployé en Alsace, l'ESV (Établissement scolaire virtuel). Marquet et Dinet (2003, 2004), croisant des observations de classe et des réponses à des questionnaires, rendent compte des utilisations privilégiées par les enseignants, celles qui renforcent leur position magistrale ; les élèves, quant à eux, ne font que consulter des informations sur Internet. Ce travail constatait l'écart important entre les potentialités offertes et les premiers usages installés et, reprenant les théorisations de Rabardel (1995), proposait une explication, pour les enseignants, en termes de difficultés d'instrumentalisation et d'instrumentation lors de l'usage simultané d'artefacts pédagogiques et d'artefacts didactiques.

\section{Des études sur le processus de déploiement}

Dans le cadre du contrat ENEIDE (Espace numérique éducatif interactif de demain), des enquêtes ont été menées par le laboratoire STEF (Sciences techniques éducation formation) dans différents établissements testant différentes solutions d'ENT (en Île-de-France et dans les académies de Strasbourg et de Nice ${ }^{31}$ ). L'objectif était de repérer des usages pédagogiques des ENT, les facteurs qui facilitent ou freinent leur utilisation et la compréhension des systèmes sociotechniques favorables à leur généralisation. Des recueils de données ont été conduits dans plusieurs lycées et collèges à dix-huit mois d'intervalle, fournissant des informations quant aux processus mêmes de déploiement au sein des établissements scolaires. Les études menées ont permis de montrer un processus de déploiement plus lent que prévu avec des établissements fragiles face à une intégration " externe " : des effets "vitrine » et des risques de rupture de dynamiques d'innovation (clairement attestés dans plusieurs établissements) et de déresponsabilisation des acteurs de terrain, faisant écho aux études menées par Collet sur le Cartable de Savoie. Les changements de logiciels et d'ENT font craindre aux enseignants l'abandon d'applications qu'ils connaissent et qu'ils considèrent adaptées à leurs besoins. En outre, les transferts d'archives (des enseignants conservent des documents qu'ils reprennent et modifient d'une année sur l'autre, ils peuvent également changer d'établissement ou intervenir sur plusieurs établissements) sont rarement assurés. Ne pas intégrer les usages existants et les innovations précédentes ne favorise pas la confiance des acteurs. 
L'implication des enseignants reste encore faible : peu d'apports originaux en classe, peu de déclarations de travail collaboratif, mais de nouveaux modes d'échange pris en charge par des enseignants innovants. Des innovations sont souvent développées avec des ENT non labellisés dans lesquels les innovateurs possèdent le contrôle sur les dispositifs qu'ils mettent en place. Cela a été bien montré par Sandrine Hénocq (en 2008) concernant les SVT (sciences de la vie et de la Terre) : les innovateurs de cette discipline qu'elle a pu rencontrer travaillaient tous avec un ENT « non officiel ». Les solutions « bricolées » sont plus ouvertes (fichiers vidéo volumineux, lien avec les téléphones portables, etc.) et contraignent moins l'inventivité des enseignants. S'ils sont aptes à trouver les astuces techniques rendant leurs projets possibles, ils n'ont pas de contrainte administrative trop forte. L'hybridation est quasiment la règle dans les établissements, c'est-à-dire l'utilisation d'autres dispositifs informatiques (logiciels, sites Internet, plateformes...) se superposant ou se substituant à celle de l'ENT. Beaucoup d'utilisateurs ont d'ailleurs des difficultés à définir et à cerner ce qu'est un ENT, en raison de la multiplicité des dispositifs existants et de la concurrence entre eux (le même service peut être assuré par une fonctionnalité intégrée à l'ENT ou par une autre application). Les confusions sont nombreuses entre l'ENT et le réseau de l'établissement, entre l'ENT et une application particulière comme le cahier de textes ou la gestion des notes, etc.

Les conditions propices au déploiement des ENT sont connues : un leadership favorable (direction et leader technique) avec une volonté claire et continue, un projet bien défini, des compétences techniques, du temps, des équipes soudées qui restent dans l'établissement... Semble attestée I'hypothèse (Coutellier, Richard, Le Loup et al., 2010) d'un déploiement plus aisé dans les établissements petits (ou à gestion plus humaine), ruraux ou semi-ruraux, avec un soutien fort de la collectivité locale, un changement visible avec l'arrivée d'un ENT susceptible de rompre un certain isolement, des communautés plus faciles à intéresser... Les facteurs défavorables à la mise en place apparaissent clairement :

- défaillances ou dysfonctionnements techniques (attribués parfois à tort aux ENT), faiblesse de l'assistance et du suivi des utilisateurs ;

- gestion délicate des annuaires, des droits et des identités, problèmes de sécurisation ;

- compétences insuffisantes des enseignants ou de l'équipe de direction : ceux qui sont compétents peuvent trouver d'autres solutions, les autres ont des difficultés à utiliser l'ENT ;

- difficultés d'accès, de conservation et de transfert ;

- crainte de perte de contrôle, de l'ingérence des parents ou du contrôle accru des inspecteurs.

Dans un travail concernant la sécurisation des accès à un espace numérique de travail, Magali Boullé (2010) a conduit cent un entretiens (dont quarante-neuf auprès d'enseignants) pour avoir l'avis des différents membres de la communauté éducative. Elle a constaté que les attentes en matière de sécurité sont différentes selon que les établissements ont installé ou non un ENT. Dans ce dernier cas, la question de la sécurisation des données personnelles est prédominante ainsi que la peur du « flicage ", tandis que dans les établissements dotés d'un ENT, bien qu'il y ait encore quelques réticences quant à son utilisation, les personnels n'ont pas de craintes concernant d'éventuels piratages du système. Les données qui sont déposées sur l'ENT ne sont pas considérées comme sensibles. Les enseignants jugent l'ENT fiable et suffisamment sécurisé, grâce notamment à l'intervention des PRI (personnes ressources informatique).

Les $\mathrm{PRI}$ jouent un rôle clé, souvent sans grande rémunération ou décharge de service. Elles assurent la médiation entre un système technique complexe et leurs 
collègues enseignants. Dépositaires de l'expertise technique dans l'établissement et porte-parole des questions pédagogiques en lien avec l'ENT pour l'extérieur, la " réussite " des ENT est en grande partie entre leurs mains. Les chefs d'établissement et les enseignants se reposent souvent sur eux. Comme l'avoue un chef d'établissement : “ Je fais une dépendance au PRI, c'est dramatique! » Que le PRI vienne à être défaillant, qu'il soit malade ou parte dans un autre établissement, et la dynamique de déploiement est en péril. Autour des aspects «vie scolaire » (gestion des absences, des notes, circulation des informations, etc.), l'ENT est souvent présenté comme un facteur de modernisation du fonctionnement des établissements. Comme le signale un PRI, « il faut être hyper carré, et en fait, un outil comme ça, s'il y a un avantage sur un ENT, enfin à la mise en place, c'est qu'il met en évidence tous les dysfonctionnements des établissements. [...] On est obligé d'être beaucoup plus carré dans les protocoles ". Toutefois, dans un autre lycée, les absences étaient déclarées par les enseignants dans l'ENT, puis imprimées et ressaisies dans un autre logiciel, montrant que la modernisation souhaitée par le ministère et les collectivités n'est pas encore vraiment effective !

Bruillard et Hourbette (2008) interrogent les modèles qui sont à la base du développement des ENT actuels, notamment l'authentification unique (SSO ${ }^{32}$ ), élément central de tous ces modèles. Si l'idée d'un mot de passe " universel » séduit certains enseignants, d'autres craignent qu'il soit le " sésame " permettant l'accès à toutes les données. Ne faudrait-il pas étudier la co-existence d'une multiplicité de possibles et de services du fait qu'il existe de nombreux autres logiciels non intégrés à l'ENT (mais cela dépend ce que l'on entend par ENT, simple intégrateur ou système d'information complet), sans compter de nombreux documents papier (carnet à souche pour l'appel et les absences, cahier de textes personnel, carnet de notes). Enfin, des détournements d'usage par les élèves ont été repérés par Danièle Hourbette, signes patents d'une appropriation, notamment le recours à des logiciels pour accéder à une messagerie instantanée ${ }^{33}$. Dans un autre établissement, une élève en fugue continuait à se connecter à l'ENT sans envoyer de message. L'ENT était-il un des derniers points de contact? Notons que des recherches sont en cours sur le cahier de textes numérique (voir par exemple Voulgre, 2011), dont l'utilisation est obligatoire pour les enseignants depuis la rentrée 2011. On peut s'attendre, concernant cette obligation, à des effets assez classiques de suivisme, de détournement, de "résistance ». Le risque, pour les chercheurs, est de trop se focaliser sur la mise en place, masquant les évolutions plus profondes liées à une diffusion largement publique d'un écrit à la circulation jadis limitée.

\section{Les parents : des acteurs symboliques?}

La mise en place des ENT soulève une nouvelle fois les questions qui se posent de manière récurrente autour de la place et du rôle des parents dans l'éducation. Le ministère, sans doute dans une volonté d'“ ouverture " des établissements scolaires, insiste beaucoup sur la place à leur donner dans les ENT. Les collectivités mettent les parents au cœur de leur action, du fait qu'elles n'ont pas de compétences en termes de programmes scolaires et de pédagogie. Le plus souvent, les recherches qu'elles sont intéressées à financer ont trait au point de vue des parents. Ainsi, une étude sur les parents a été effectuée par Nacira Aït-Abdesselam (2009), dans le cadre des travaux menés par le laboratoire CIVIIC (Centre interdisciplinaire de recherches sur les valeurs, les idées, les identités et les compétences en éducation et en formation) dans le département des Hauts-de-Seine (Daguet \& Voulgre, 2011 ; Voulgre, 2011). Cette étude montrait une adhésion et une mobilisation des parents sur le projet ENT, qui concourait à les rassurer. Mais cette adhésion s'inscrivait plus dans 
une logique de contrôle de leur enfant que dans une logique de collaboration avec l'école.

Bruillard et Hourbette (2008) constatent une implication encore embryonnaire des parents et l'apparition de tensions, confirmée par plusieurs rapports de l'inspection générale de l'Éducation nationale sur les ENT ou la politique concernant les TICE (Technologies de l'information et de la communication en éducation). En particulier, une gestion de la vie scolaire uniquement médiée par l'ENT (risquant de diminuer le nombre des contacts personnels avec les parents) est loin de faire l'unanimité, et la question de la gestion et de la mise en ligne des notes est problématique. Des réticences, justifiées par les risques d'inégalité, voire d'exclusion de certains parents, sont exprimées par les enseignants également inquiets face aux possibilités de comparer les cours une fois mis en ligne et de critiquer leurs choix pédagogiques. Toutefois, les fédérations de parents d'élèves semblent encore peu concernées, puisque rien concernant les ENT n'est visible sur le site national de la FCPE (Fédération des conseils des parents d'élèves), sauf un dossier sur le poids du cartable, rien sur le site de la PEEP (Fédération des parents d'élèves de l'enseignement public).

\section{Que disent les syndicats d'enseignants et de chefs d'établissement ?}

Même s'il ne s'agit pas de recherche, le point de vue exprimé par les différents syndicats d'enseignants est une des pièces du puzzle constitué par le déploiement des ENT. Un premier travail mené par Cécile Ferlin en 2008 n'a pas été très concluant quant à leur intérêt pour le déploiement des ENT. Semblant attendre que les utilisations se développent, les syndicats ne souhaitaient pas soulever les problèmes à l'avance. En revanche, en 2009, un débat très animé a émergé et un front de résistance s'est constitué contre la constitution de fichiers nominatifs concernant les élèves (fichier "Base élèves »), jusqu'à obtenir un arrêté du Conseil d'État pour l'obtention d'un droit d'opposition ${ }^{34}$.

Les syndicats commencent, en 2011, à prendre des positions. Pour résumer, on peut dire que le SGEN (Syndicat général de l'Éducation nationale) était plutôt pour le déploiement des ENT, tout en reconnaissant les difficultés rencontrées ${ }^{35}$, mais a exprimé son opposition à l'idée d'un « déploiement industriel d'ENT conçus comme des outils d'administration et de contrôle 36 ". Le SNES (Syndicat national des enseignants du second degré) a produit une brochure informative ${ }^{37}$ rappelant la législation, notamment sur le cahier de textes, sur la liberté pédagogique ou sur la protection de la vie privée. Certains sites du SNES interrogent la pertinence des TIC en éducation et mettent en garde sur le rôle que peut jouer l'ENT dans un pilotage accru par le local et sur une possible privatisation des contenus et des outils pédagogiques ${ }^{38}$. Des sections de SUD Éducation (Solidaires unitaires démocratiques) alertent sur un aspect Big Brother des ENT, des fichiers personnels qu'ils constituent pouvant être utilisés à des fins de contrôle ${ }^{39}$. Le SNPDEN, syndicat des chefs d'établissement, confirme une prise en compte insuffisante des besoins des établissements et des difficultés de gestion en $2007^{40}$, interrogations que l'on retrouve en 200941 , montrant des inquiétudes quant à la concertation, à la cohérence et aux déficits en personnels, en formation, en assistance et en maintenance. 
Le tour d'horizon précédent montre une recherche active, révélant certains points d'achoppement, permettant de mieux comprendre un système complexe en devenir. Plus précisément, la recherche atteste d'un processus de déploiement des ENT lent, non linéaire, d'une appropriation encore limitée, de dysfonctionnements techniques. Elle montre que des problèmes non anticipés émergent peu à peu. Alors qu'un consensus se dégage de ces recherches, ainsi que des comparaisons internationales (voir plus haut), sur l'importance de faire participer les enseignants depuis la conception, le plus en amont possible, les processus à l'œuvre sont très différents. Le caractère affiché comme inéluctable d'un déploiement industriel des ENT, infrastructure jugée indispensable à la modernisation du système éducatif, tranche avec des résultats de terrain indiquant un professionnalisme « limité ». On n'anticipe pas les problèmes des acteurs, mais plutôt les questions d'aménagement (haut débit, fibre optique, etc.) et l'ENT est avant tout un élément dans des processus plus complexes concernant les territoires et leurs relations avec l'État.

Les recherches que nous venons d'exposer adoptent le plus souvent une approche systémique, elles sont plus proches des sciences de l'information que des sciences de l'éducation (les questions proprement éducatives n'y apparaissent qu'en toile de fond). La mobilisation de la théorie de l'acteur réseau pour étudier les déploiements des projets ENT semble pertinente et productive. Cette perspective de recherche n'est toutefois pas reprise par les institutions de pilotage. Concernant ces dernières, bien que l'on ne connaisse pas les modèles susceptibles de sous-tendre leur action, les discours et écrits officiels en donnent des traces suffisamment explicites pour que l'on puisse y déceler quelques idées sous-jacentes et montrer comment des recherches peuvent aider à les déconstruire. Le modèle de pensée est simple, voire simpliste et peut être décrit grossièrement : il y a un processus linéaire de diffusion ou de "généralisation des usages ", on peut catégoriser les enseignants vis-à-vis de ce processus et il s'agit de mettre en œuvre des formes de transformation pour faire passer les enseignants d'une catégorie à l'autre. Autrement dit, c'est d'abord l'idée d'“ intégration " qui domine les discours, qui va ensuite convoquer les recherches de bonnes pratiques et proposer la mise en œuvre de modèles managériaux de conduite du changement. Mais la première chose, c'est la mise à l'écart des questions de technique, que l'on cherche à éloigner des préoccupations de l'enseignant.

\section{Un déni : la technique ; une illusion : la transparence}

Masquer la complexité technique permettrait aux utilisateurs de " se concentrer sur les apports des TIC pour la pédagogie et l'organisation de l'établissement d'enseignement 42 ". S'agissant d'apprentissage, l'idée qu'une technologie puisse gérer complètement les aspects « calculatoires » permettant de se concentrer sur des points de vue plus conceptuels et ainsi de mettre les élèves en situation de recherche, a plusieurs fois été démentie, notamment en EXAO43 ou avec l'utilisation des calculatrices en mathématiques (Bruillard, 1997). Jusqu'où peut-on masquer des aspects techniques et quel est l'effet de ce masquage ? Peut-on maîtriser des fonctionnalités masquées ? Évacuer les questions techniques, n'est-ce pas la meilleure façon de rendre les enseignants dépendants, esclaves des techniques et des pouvoirs qui les maîtrisent ? Si une opposition entre des approches qualifiées de technocentrées et anthropocentrées (Rabardel, 1995) n'est pas dénuée de fondement, elle court le risque d'opérer un clivage finalement peu opératoire, cachant les imbrications entre les questions techniques et les questions plus proprement humaines. Or ce qui est 
important, ce sont précisément les interactions entre les univers sociaux et les univers techniques, entre les acteurs humains et les objets construits, etc. Malgré cela, subsiste une opposition simpliste entre technique et pédagogie ou didactique.

La manière dont les autorités éducatives présentent le déploiement des ENT joue sur la confiance que devrait donner l'infrastructure technique. «En assurant la sécurité des données manipulées, la simplicité des accès (une seule authentification) et la couverture des besoins en fonction des profils d'usagers, les ENT constituent un cadre de confiance pour l'utilisation des outils et des ressources numériques dans les établissements " (MEN, 2010, p. 13-14). Les mots-clés " sécurité », " simplicité ", " couverture ", « confiance » caractérisent un mode de pensée. S'adresse-t-il vraiment aux enseignants ? N'est-il pas plutôt à destination des parents, faisant de l'école un lieu protégé et sécurisé ? En tout cas, cela conduit les enseignants à adopter une position de consommateurs et la technique va finalement aider à normaliser les pratiques, relayer les prescriptions. Dire qu'enseigner est une activité instrumentée, c'est dire qu'il faut la penser avec les instruments, que l'on ne peut pas facilement les dissocier de l'activité. Or, les discours sur l'intégration, sur la technique, consistent à en faire une entité séparée que l'on va «importer » et « intégrer " ; il n'y a pas de vision (explicitée) d'une transformation plus complexe liée à l'évolution de l'instrumentation associée.

\section{Des modèles d'intégration des technologies en éducation fondés sur des théories diffusionnistes et des oppositions réductrices}

Dans le domaine des technologies en éducation, à la notion d'intégration peut être préférée celle de scolarisation, mettant mieux en lumière des processus dont l'enjeu est «l'appropriation collective et la légitimation progressive de nouveaux instruments dans des activités finalisées " (Baron \& Bruillard, 2004, p. 160). La forme scolaire va être transformée par les technologies mais va également les transformer. Des principes d'économie et d'efficience vont guider l'appropriation individuelle et des considérations de compatibilité et de légitimité vont être au cœur de l'appropriation collective.

L'intégration correspond au point de vue du pilote, celui de l'institution, et va de pair avec le développement des modèles de gestion. Les modèles d'intégration vont décrire des paliers, des phases, des états, fournir des listes de critères pour juger de l'état d'avancement des établissements et parfois proposer des pistes pour faciliter les transitions entre les états. Ces modèles par étapes sous-entendent souvent un état final peu questionné ; les changements des technologies sont passés sous silence ; des étapes, vite pensées comme nécessaires, conduisent à des catégorisations des acteurs (des innovateurs aux résistants). D'abord présents dans les pays développés, quand l'intérêt politique diminue, ils se diffusent à l'UNESCO pour les pays émergents. Mais leur validité et leur puissance explicative sont loin d'être attestées.

En fait, les modèles d'intégration ne sont pas discutés vis-à-vis des modèles d'innovation technologique dans l'éducation, mais sont peu ou prou compatibles avec le modèle diffusionniste de Rogers (1995). Ce dernier décrit des phases selon lesquelles vont être adoptées les innovations et surtout propose une catégorisation des usagers en les plaçant sur une échelle temporelle de la diffusion : les innovateurs, les adoptants précoces, la majorité précoce, la majorité tardive, et les retardataires (Rogers, 1995, p. 22). Jauréguiberry (2010) rappelle que le modèle de Rogers postule que l'innovation est positive et que la problématique, concernant la majorité tardive et les retardataires, consiste à " surveiller le degré d'acceptation du milieu 
réceptif qui l'adoptera plus ou moins rapidement ". "Par son côté normatif, facile à comprendre et semblant tomber sous le sens " (Jauréguiberry, 2010, p. 23), ce modèle est facilement adopté. II a été perfectionné, mais "l'essentiel a consisté à cerner les facteurs qui freinent et contrarient l'adoption des innovations " (Jauréguiberry, 2010, p. 24). Bien évidemment, les industriels vont financer de multiples études empiriques permettant de mieux cerner les points de résistance ou de blocage et mettant en œuvre des techniques statistiques sophistiquées afin de cerner la ou les variables supposées expliquer les écarts constatés.

Poursuivre une recherche ayant simplement pour rôle de mesurer des écarts et d'identifier les résistances risque dès lors de produire des données empiriques renforçant des cadres théoriques non questionnés. Les modèles de catégorisation donnent un cadre interprétatif qui fonctionne à tous les coups : on trouvera toujours des adoptants précoces et des retardataires, ce qui ne valide en rien un processus linéaire de diffusion. Les mêmes problèmes et présupposés apparaissent dans les modèles explicatifs de l'acceptation des TIC et dans ceux qui visent l'accompagnement face à des changements organisationnels. Une telle direction de travail consiste à faire reposer sur les sujets individuels le poids d'une acceptation ou d'une résistance vis-à-vis des technologies. En fait, on les sépare de leurs attaches collectives, dans une vision peu éclairée du métier d'enseignant.

\section{Identifier les facteurs d'acceptation des technologies informatiques : des modèles en question}

Essayer d'identifier les facteurs principaux qui expliquent l'acceptation d'une application informatique, mais prédire également le poids relatif de chacun d'entre eux, ce sont les objectifs que se donnent des modèles généraux comme le TAM (technology acceptance model) de Davis $(1985,1993)$ ou I'UTAUT (unified theory of acceptance and use of technology), de Venkatesh, Morris, Davis et alii (2003).

Le modèle TAM vise à fournir une base pour découvrir l'impact de variables externes sur des variables internes : croyances, attitudes et intentions (Marchewka, Liu \& Kostiwa, 2007). II rend compte du processus d'acceptabilité d'un système grâce à deux facteurs principaux : son utilité et sa facilité d'usage telles qu'elles sont perçues par les utilisateurs. Selon les auteurs du TAM, ces deux facteurs influencent leurs attitudes, lesquelles influencent leur intention d'utiliser la technologie en jeu. Une très abondante littérature est disponible sur ces modèles, principalement en sciences de la gestion, où l'on cherche aussi à développer des outils d'intervention afin d'accroître (améliorer) l'acceptation (Bourdon \& Ollet-Haudebert, 2009) mais également en ergonomie. Dans ce dernier cas, un objectif est, selon Humbert (2010), d'aider les concepteurs, de mieux leur faire connaître les contextes de réception afin de réduire l'incertitude concernant l'appropriation de leurs produits par les futurs usagers. En gestion, le but est souvent d'aider des entreprises mettant en place de nouveaux outils à identifier les stratégies à adopter (par exemple en Chine, voir Venkatesh \& Zhang, 2010).

Ces modèles commencent à être utilisés avec les ENT à l'université (Billouard, 2011) ou dans le secondaire (Schneewele, Cherqui-Houot, Nowakowski et al., 2010 ; Pacurar, Abbas \& Moltini-Zender, 2011). Pourtant, leur fiabilité est actuellement très discutée. Des études précises ont été menées en psychologie ergonomique en France.

Souhaitant développer un outil francophone d'évaluation des intentions d'usage intégrant notamment la dimension émotionnelle, Février, Jamet et Rouxel (2008) ont mis à l'épreuve le TAM. Ils ont évalué les intentions d'utiliser l'ENT de l'université 
Rennes 2, auprès de cent quatre-vingt-douze nouveaux inscrits à l'université et n'ont pas retrouvé la structure factorielle du modèle original. Le même résultat a été obtenu avec deux autres études sur la découverte de nouveaux systèmes, les conduisant à émettre un doute quant à la robustesse du modèle. Plus récemment, Février, Gauducheau, Jamet et alii (2011) rendent compte d'autres critiques portant sur le modèle TAM, citant les travaux de Brangier, Dufresne et Hammes-Adelé (2009). Le modèle souffrirait d'insuffisances méthodologiques : d'abord, les résultats publiés présentent beaucoup de variabilité voire sont parfois contradictoires ; le pouvoir explicatif du TAM est ensuite relativement limité et sa structure factorielle n'est pas systématiquement répliquée ; il véhicule enfin une conception plutôt rationnelle et déterministe de l'acceptation (et donc de l'individu). Février, Gauducheau, Jamet et alii (2011) préconisent de mieux prendre en compte les côtés émotionnels dans l'expérience des utilisateurs avec les technologies et l'expression « approche symbiotique de la relation humain-technologie » de Brangier, Dufresne et Hammes-Adelé (2009) témoigne d'un point de vue très éloigné de celui du TAM. Au-delà de la discussion sur la fiabilité de ces modèles, on peut s'interroger sur le bien-fondé de leur utilisation ; il n'est pas très crédible de faire tester un ENT, intégrateur de services, par des utilisateurs potentiels et de mesurer leur motivation à l'utiliser de manière plus étendue. Mais le recours au modèle TAM est peut-être compatible avec le processus de déploiement des ENT, tel qu'il est conçu actuellement. En continuation avec le modèle de Rogers, la question des "retardataires " va se traduire par la résistance au changement, dans un modèle résolument descendant d'innovation.

\section{La résistance au changement : vers une vision plus positive des non-usagers?}

La rhétorique de la résistance au changement est classique dans les discours portant sur l'intégration des technologies dans l'éducation et plus largement quand il s'agit d'innovations institutionnelles. Cette idée de prendre en compte une supposée "résistance au changement " est présente dans les textes présentant le schéma directeur des ENT : “ Un projet ENT pris dans son ensemble a un impact général sur la transformation d'un établissement. La résistance au changement et surtout le poids des habitudes sont deux risques majeurs à prendre en considération. [...] Avec la mise en place d'un ENT, les approches comportementales doivent elles aussi changer. Les enseignants deviennent en quelque sorte porte-parole du SDET et incitent les élèves à s'appuyer sur ce nouvel outil dans le cadre de leur apprentissage. Le personnel administratif peut se faire le relais auprès des parents quant à la gestion de la scolarité de leurs enfants. Et ainsi de suite 44 ". Dans la phase de déploiement dans les établissements, il s'agit de transformer d'éventuels résistants au changement en agents de changement.

Des revues de questions, menées en sciences de gestion, permettent de situer cette notion de « résistance au changement ". Selon Cécile Bareil (2004), " aucun fondement n'a pu être démontré au sujet des résistances au changement, sauf une acceptation presque aveugle de la communauté scientifique à l'égard du phénomène ", et l'angle de la résistance au changement pour l'étude du changement a été préjudiciable à la recherche. ljaz et Vitalis (2011) observent que la résistance a cessé d'être considérée uniquement comme un processus psychologique interne déviant. Produit des interactions entre un agent et un destinataire du changement, elle peut être vue comme l'interprétation de cet agent de changement sur la réaction du destinataire. Ford, Ford et D'Amelio (2008), dans une publication au titre évocateur (Resistance to change: The rest of the story), montrent que les agents de chan- 
gement contribuent à l'émergence de la résistance à travers leurs propres actions et inactions et que la résistance peut être une ressource pour le changement.

Par ailleurs, selon Meissonier, Houze et Belbaly (2007), si la résistance des utilisateurs face à l'implantation des TIC est un thème majeur dans le domaine des systèmes d'information, elle n'a pas forcément été traitée suffisamment en profondeur. Ils citent en appui un article de Lapointe et Rivard (2005), révélant que « sur quarante-trois des plus prestigieux articles publiés sur le sujet au cours des vingt dernières années, seulement quatre ne se limitaient pas à aborder la résistance des utilisateurs comme un élément factuel, et ouvraient la "boîte noire" en tentant d'en découvrir les causes et les formes ".

On peut légitimement s'interroger sur le bien-fondé de cette thématique de la résistance aux TIC dans le système scolaire. On pourra toujours accoler une étiquette de résistant à différents acteurs, sans y attribuer une signification bien claire. Finalement, qui sont les non-usagers et pourquoi le sont-ils ? Comment expliquer le non-usage : comme un manque, un aspect négatif, un trou à combler ? Serait-il passif, subi ? Les travaux menés en sociologie des usages sur les non-usagers (Jauréguiberry, 2010 ; Kellner, Massou \& Morelli, 2010) montrent un côté positif et actif du non-usage. Daguet et Voulgre (2011) ont recueilli des déclarations de nonusages ou de faibles usages qu'ils qualifient de revendiqués, la cause liée à la méconnaissance n'apparaissant pas la plus développée. Un autre aspect rencontré est celui du désengagement : des enseignants qui ont commencé à utiliser puis ont renoncé, souvent suite à un changement de système informatique. N'est-ce pas avec ces prétendus « résistants » que pourrait se construire un usage raisonné des TIC en éducation ? Ainsi, Cacheux (2009) s'appuie sur des entretiens de décideurs (qu'elle étudie à l'aide de l'analyse conversationnelle) et sur l'observation d'enregistrements audiovisuels de séances de formation d'enseignants à l'ENT, pour étayer son hypothèse selon laquelle les acteurs participent activement par leur " résistance " à l'évolution des usages dans un processus d'innovation en cours, citant Anne-Marie Laulan et la résistance aux systèmes d'information (Laulan, 1985).

\section{Quelle place pour les enseignants ?}

En l'absence d'une vision claire sur le fonctionnement de l'école à l'issue du processus actuel de généralisation des ENT et de sur-technologisation, les enseignants peuvent avoir des interrogations. Rappelons la double contrainte à laquelle ils sont classiquement soumis, comme l'a bien montré Larry Cuban (1986). D'un côté, ils doivent être modernes et pour cela s'avérer utilisateurs des dernières technologies apparues qui se diffusent dans la société, mais, d'un autre côté, il faut qu'ils contrôlent du mieux possible les apprentissages de tous les élèves présents dans leur classe. Cette dernière injonction est bien évidemment plus essentielle que la précédente. Elle constitue le cœur de leur mission et, le plus souvent, les technologies, s'ils ne les maîtrisent pas suffisamment, compliquent leur travail.

Des innovateurs proches de la technique jouent un rôle très important dans le système éducatif : ils testent les derniers environnements et matériels, en inventent des utilisations pédagogiques. Ils sont en phase avec les " marchands ", puisqu'ils vont aider à trouver des issues éducatives aux technologies proposées, et avec une partie des autorités éducatives qui peuvent afficher une garantie de réactivité du système scolaire. Ce sont souvent ces innovateurs qui inventent les situations qui vont se scolariser. Mais ils sont condamnés à se renouveler constamment, pour rester en phase avec les dernières offres techniques. Ils acquièrent un statut au sein du système éducatif, mais ce statut est en général plutôt précaire. De tels bricolages 
sont nécessaires, articulés avec l'innovation ordinaire (Alter, 2000) que réalisent nombre d'enseignants. Mais jusqu'où sont-ils compatibles avec les ENT une fois ceux-ci entièrement déployés ? On peut raisonnablement penser que les acteurs conserveront une marge d'initiative, mais les contraintes risquent de s'alourdir.

Ainsi, l'expérimentation nationale « Manuels numériques via l'ENT » avait officiellement pour objet d'aider à développer des utilisations innovantes des manuels numériques, tant en classe qu'à la maison pour les élèves ${ }^{45}$. Dès la première année, en raison de différents problèmes, notamment techniques, l'expérimentation s'est transformée en un projet centré sur les utilisations en classe, via les tableaux numériques interactifs pour l'essentiel, renforçant et améliorant une pédagogie de la « monstration » des enseignants. Pour la seconde année, le ministère reconnaît un nombre beaucoup plus important de dysfonctionnements techniques et confirme une faible utilisation par les élèves ${ }^{46}$. Toutefois, les manuels et ressources qui ont le mieux fonctionné, tant en classe qu'à la maison, sont ceux développés par l'association d'enseignants Sésamath ; les difficultés techniques constatées confirment une fois encore que le côté industriel revendiqué tarde à faire la preuve de sa supériorité. Un problème ne viendrait-il pas en partie du poids économique des grands éditeurs scolaires et de leur difficulté à trouver un modèle économique viable, conduisant à contraindre le fonctionnement technique des ENT pour l'accès aux ressources éducatives et pour leur gestion?

En effet, les technologies conduisent à une évolution économique importante : le passage de la possession de biens à la location de services. Cette transformation touche également l'éducation et les éditeurs scolaires souhaitent conserver leur marché. Devenant un service, le manuel numérique risque de n'être accessible que pendant la durée d'un contrat, il ne sera plus la propriété des établissements, l'ENT devenant à la fois le moyen d'accès, le compteur des utilisations et le garant de cette utilisation. Mais, pour les éditeurs scolaires, il faut éviter que les enseignants puissent récupérer les ressources afin de les recomposer à loisir pour les adapter à leur classe, en contournant l'ENT.

La réussite de l'association Sesamath dans la production de ressources comme, dans une moindre mesure, de Sesâme, Weblettres ou des Clionautes 47 , atteste que l'innovation horizontale ou ascendante (Von Hippel, 2005 ; Cardon, 2006) peut constituer un modèle alternatif possible et que les communautés d'enseignants pourraient peut-être prendre en charge plus largement la conception, la production et l'échange de leurs ressources éducatives.

\section{PERSPECTIVES}

Les difficultés pointées par Henri Dieuzeide (1982), la tension entre des offres techniques extérieures à un système éducatif et souhaitant l'investir sans l'écouter, ne rendent qu'imparfaitement compte de la situation actuelle. Les ENT étaient censés répondre à des problèmes bien repérés, mais la mise en œuvre des " solutions " conduit à un renversement et à imposer aux acteurs de l'éducation une infrastructure constituant leur cadre de travail48. Les TIC interviendraient comme un facteur clé de modernisation du système éducatif. Cependant, pour certains experts américains, les systèmes scolaires des grands pays industrialisés ne peuvent pas « intégrer » les TIC de manière significative. Systèmes robustes, adaptés à une société industrielle, ils n'ont pas la capacité d'ajouter les TIC, trop en décalage, dans leur économie actuelle. Ces auteurs prédisent des évolutions importantes à plus ou moins brève échéance, 
avec des effets sociaux dont il faudrait rapidement prendre la mesure (Collins \& Halverson, 2009 ; Christensen, Horn \& Johnson, 2008). Le système scolaire français ne suivra pas forcément les modèles américains, mais il semble sommé d'évoluer et de se réinstrumenter. Ce qui est en question est une "re-technologisation ", ou plus exactement une réinstrumentation de l'école, à certains égards, similaire à la " redocumentarisation » (Salaün, 2008). Le processus de déploiement des ENT est caractéristique des systèmes d'information englobants qui se développent depuis plusieurs années. Dans leur mise en place, avec une rationalisation à outrance, l'usager en tant que personne disparaît derrière un profil et des besoins qui lui ont été attribués. Yves Chevalier (2008), pour les ENT dans les universités, montre de manière convaincante la fin de l'usager face aux systèmes d'information globale qui se déploient, ne laissant place qu'à des usages légaux ou délictueux.

Quelle recherche est possible, dans un système éducatif en mutation et face à de multiples parties prenantes (académies, collectivités) ? Comment faire évoluer un modèle de recherche national avec des exigences internationales, sans devenir des prestataires pour des études et des accompagnements au gré des commanditaires locaux ? La recherche ne peut être dans la droite ligne d'un discours officiel de déploiement linéaire et continu. Trop ethnographique, elle risque de passer pour anecdotique. Attirée par ce qui émerge de différent, par les problèmes qui surviennent, elle n'est pas du goût des prescripteurs. Les recherches à l'horizon trop limité ne peuvent être qu'instrumentalisées par les acteurs institutionnels ou disqualifiées parce que perçues comme trop critiques ou trop négatives. Ce qui manque, ce sont des débats, des mises en discussion de multiples options dans la compréhension des phénomènes liés aux ENT. Une recherche pluridisciplinaire s'avère nécessaire, prenant en compte de multiples facteurs, dans des approches ouvertes pour articuler global et local, pour problématiser au plan local dans le respect de contraintes qui nous échappent, afin d'identifier les problèmes, de proposer des analyses globales, de faire des cartographies, de fournir des cas d'étude. La recherche est peu présente et le milieu ou plutôt les milieux de recherche sont trop faibles, trop émiettés pour soutenir et légitimer des discours forts. Elle devrait mieux s'affirmer. C'est un travail qui devrait se conduire avec les enseignants et avec les cadres du système éducatif et des collectivités, dans une responsabilité partagée.

Éric Bruillard eric.bruillard@ens-cachan.fr ENS Cachan, STEF

NOTES

1 Séminaire à l'ENS Cachan, en ligne : http://www.stef.ens-cachan.fr/manifs/sem_ens_tic/index.html.

2 En ligne: http://www.caissedesdepots.fr/le-groupe/qui-sommes-nous/un-investisseur-unique.html.

3 En ligne : http://www.caissedesdepots.fr/activites/accompagner-les-collectivites-territoriales/lesinvestissements-dinteret-general/domaines-dintervention.html.

4 En ligne: http://www.cyber-base.org/institutionnel/univers/homeProgramme.do.

5 En ligne: http://www.cyber-base.org/institutionnel/home.do.

6 Commission nationale de l'informatique et des libertés. En ligne : http://www.cnil.fr/.

7 Arrêté du 30 novembre 2006 portant création, au sein du ministère de l'Éducation nationale, de l'Enseignement supérieur et de la Recherche, d'un traitement de données à caractère personnel relatif aux espaces numériques de travail (ENT, voir NOR : MENT0602397A).

8 En ligne : http://projets-ent.com/ressources/mode-demploi-de-la-generalisation/quest-ce-quunespace-numerique-de-travail/.

9 En ligne : http://www.educnet.education.fr/services/ent/scolaire/defi-ent, consulté le 31 juillet 2010 , avec la date de publication : 2 décembre 2009 (consulté le 30 juillet 2010). 
10 En ligne : http://eduscol.education.fr/cid57080/historique-des-ent.html (consulté le 27 novembre 2011).

11 En ligne : http://www.education.gouv.fr/cid382/10-mesures-pour-relancer-l-utilisation-des-technolo gies-de-l-information-et-de-la-communication-a-l-ecole.html. Luc Ferry et Xavier Darcos : " D'ici 2007, chaque élève, chaque enseignant et chaque famille disposeront d'un accès personnel à son espace d'information et de travail, conformément aux objectifs du plan RESO ", en ligne : http://www. educnet.education.fr/textes/discours/2003.htm.

12 En ligne: http://www.education.gouv.fr/cid20945/environnement-numerique-de-travail-xavier-darcosdans-l-academie-de-toulouse.html.

13 La Fondation pour l'Internet nouvelle génération (FING) est un think tank créé en 2000 par une équipe d'entrepreneurs et d'experts, dont l'objet est d'anticiper les transformations numériques. Cette association produit notamment des rapports pour les entreprises et les collectivités. En ligne : http://fing. org/?-Presentation-. Le rapport cité est disponible sur le site de l'ARTESI, agence de développement des TIC associée au conseil régional d'île-de-France.

14 Schéma directeur des ENT. En ligne : http://eduscol.education.fr/cid56994/preconisations-techniques. html.

15 En ligne: http://projets-ent.com/cartographie-2/carte-interactive-des-ent/.

16 En ligne: http://eduscol.education.fr/cid55728//-etat-du-deploiement.html.

17 En ligne : http://eduscol.education.fr/bd/etic/2011/lycees_2011_11_07l.htm.

18 En ligne: http://preprod.landesinteractives.net/default.asp.

19 En ligne : http://www.educnet.education.fr/services/ent/scolaire/enjeux-ent.

20 En ligne : http://www.educnet.education.fr/services/ent/scolaire/Certification_ENT.

21 En ligne : http://eduscol.education.fr/pid25780-cid55740/dispositifs-d-evaluation-des-usages-desent.html.

22 Observatoires des territoires numériques (http://www.oten.fr/). En ligne : http://www.oten.fr/spip. php?article4231.

23 En ligne : http://www.valoffre.caissedesdepots.fr/IMG/pdf/ENT_CDC_Synthese_etude_10p_v2.pdf. Cette étude a été menée par le cabinet Sagatel, pour le compte de la Caisse des dépôts et consignations et en partenariat avec le ministère de l'Éducation nationale.

24 En ligne : http://projets-ent.com/ressources/les-plates-formes-virtuelles-d-apprentissage-en-europe/ facteurs-de-succes-2/.

25 Selon une étude menée en 2005 par le cabinet Accenture, les facteurs les plus significatifs de gain concernent la dématérialisation des échanges (envoi des relevés de notes, des relevés d'appels à pension, des relances, des notifications d'absences, et d'informations relatives à la vie des établissements). L'adoption généralisée de l'ENT par les parents est pour cela indispensable. ARF, CDC, Accenture, Étude du modèle économique de l'ENT et de l'impact pour les régions de sa généralisation. Synthèse de l'étude. En ligne : http://www.valoffre.caissedesdepots.fr/IMG/pdf/ENT_CDC_Synthese_ etude_10p_v2.pdf (consulté le 18 février 2008). Notons que ce rapport n'est plus disponible. II est présenté par Nicolas Monsarrat dans un séminaire à Cachan (http://www.stef.ens-cachan.fr/manifs/ sem_ens_tic/CDC_ENS_TCO_Monsarrat.pdf). II semble que l'étude ait été conçue dans le but de convaincre les collectivités locales d'investir dans les ENT. Toutefois, on peut constater que les établissements scolaires disposant d'un ENT sont confrontés à la nécessité de continuer à doubler les envois électroniques par des envois papier, conduisant plutôt à une augmentation des coûts qu'à une diminution.

26 Appel à projets pour la mise en place d'études territoriales sur les usages des espaces numériques de travail du second degré. En ligne : http://www.valoffre.caissedesdepots.fr/lMG/pdf/Appel_a_projet etudes_des_usages_ENT_2011.pdf. II semble que peu de collectivités se soient intéressées à cet appel et que l'incitation à la recherche lancée par la CDC ait eu peu d'effet.

27 En ligne : http://www.stef.ens-cachan.fr/ent/ent_journee_rech_11062008.htm et http://www.stef.enscachan.fr/ent/ent_journee_rech_11032010.htm.

28 En ligne : http://eductice.inrp.fr/EducTice/projets/en-cours/usages/ouvre.

29 En ligne : http://eductice.inrp.fr/EducTice/projets/en-cours/usages/apparent.

30 Notons le travail de Lamia Badra visant à reconstruire les univers de référence des acteurs impliqués dans le pilotage et le développement de l'ENT d'Auvergne. En ligne : http://www.stef.ens-cachan.fr/ ent/ent_badra_11_mars_2010.pdf.

31 Travail que j'ai dirigé avec François-Marie Blondel. Les études sur le terrain ont été conduites par Danièle Hourbette, Cécile Ferlin, Emmanuelle Voulgre et Magali Boullé.

32 Single sign on, le fait que les utilisateurs n'ont à s'authentifier qu'une seule fois, par exemple en saisissant un nom et un mot de passe, pour avoir accès à toutes les informations et tous les services associés à leur profil.

33 Utilisation de Meebo : en ligne, http://www.meebo.com/, ou de Imo.im, en ligne : https://imo.im/.

34 Voir en ligne : http://blog.dalloz.fr/2010/09/big-brother-sinvite-dans-les-cours-decoles/ et http:// retraitbaseeleves.wordpress.com/2011/06/29/region-paca-contre-les-fichiers-scolaires/.

35 En ligne: http://blog.sgen.net/numerique/? $p=144$.

36 En ligne : http://www.cfdt.fr/content/medias/media33377_gQbndPqDjDhcWTn.pdf. 
37 En ligne : http://www.snes.edu/IMG/pdf/Point_sur_les_TICE_et_les_ENT_sept_10-2.pdf.

38 En ligne: http://www.snes.edu/Les-Environnements-numeriques-de.html.

39 Voir notamment, en ligne : http://sudeducvendee.ouvaton.org/spip.php?article8.

40 Dans Direction, no 154 (de décembre 2007) est présentée une motion à un congrès interne : « Le pilotage de l'EPLE [établissement public local d'enseignement] nécessite un espace numérique de travail performant qui doit être conçu à partir des besoins exprimés par les EPLE. " Et plus loin, attestant les difficultés dans la gestion, est revendiquée "l'élaboration d'un cahier des charges cohérent définissant clairement la responsabilité de l'État et des collectivités territoriales dans la maintenance des équipements et la gestion des réseaux pédagogique et administratif ». En ligne : http://www.snpden. net/direction/pdf154/page44_48.pdf.

41 «Si l'environnement numérique de travail est devenu utile au pilotage des EPLE, sa mise en œuvre et la non-pertinence de certaines applications informatiques complexifient, voire alourdissent, le fonctionnement quotidien de nos établissements. Les développements disparates des ENT font apparaître aujourd'hui la nécessité de définir un cahier des charges national, académique et départemental garant d'une indispensable cohérence. Enfin, la mise en œuvre des ENT ne peut se concevoir sans la présence en EPLE de personnels qualifiés et en nombre suffisant, sans formation des utilisateurs à l'usage des applications et sans un service d'assistance et de maintenance du matériel informatique répondant aux besoins. Les rapports de l'EPLE avec l'État et les collectivités territoriales doivent être basés sur la concertation, la contractualisation et le conventionnement, et non sur la sujétion et l'injonction. 》 En ligne : http://www.snpden.net/node/313.

42 En ligne : http://www.educnet.education.fr/services/ent (consulté le 31 juillet 2010, date de publication : 2 avril 2010).

43 EXAO : expérimentation assistée par ordinateur. Brigitte Vialle (2001) a montré qu'avec l'EXAO, les élèves se ressentent éloignés des phénomènes biologiques qu'ils sont censés étudier et se perçoivent parfois comme servants d'un système technique qui décide à chaque moment ce qui est possible, voire ce qui est souhaité.

44 Dans le schéma directeur SDET 2.0 du 7 novembre 2006, p. 65, consulté le 31 juillet 2010 à l'adresse : ftp://trf.education.gouv.fr/pub/educnet/chrgt/sdet/SDET_v2.0.pdf.

45 Voir en ligne : http://www.educnet.education.fr/contenus/fichiers/fichiers-manuel-numerique/expemn-dispositif-evaluation (consulté le 15 juillet 2011).

46 Voir en ligne : http://eduscol.education.fr/cid58607/experimentation-manuels-numeriques-via-l-entdes-resultats-relativement-positifs-pour-la-seconde-annee-d-evaluation.html.

47 Associations d'enseignants de mathématiques (en ligne : http://www.sesamath.net/), d'histoire et géographie (http://www.clionautes.org/), de lettres (http://www.weblettres.net/), de sciences économiques et sociales (http://sesame.apses.org/) produisant des ressources éducatives et même, pour Sésamath et Sesâme, des manuels scolaires.

48 Notons toutefois l'engouement actuel pour les tablettes numériques, en quelque sorte versions actuelles du bureau virtuel ou du cartable électronique.

\section{BIBLIOGRAPHIE}

AÏT-ABDESSELAM N. (2009). Sens et enjeux de l'ENT : les usages des parents d'élèves de quatre collèges des Hauts-de-Seine. Note d'étape sur le dispositif ENC 92, rapport remis au conseil général des Hauts-de-Seine. En ligne : http://www.stef.ens-cachan.fr/ent/ ent_ait_abdesselam_11_mars_2010.pdf (consultée le 15 décembre 2011, comme les autres documents de cette bibliographie, sauf mention contraire).

AKRICH M., CALLON M. \& LATOUR B. (2006). Sociologie de la traduction : textes fondateurs. Paris : Transvalor et Presses des Mines.

ALBERO B. \& THIBAULT F. (2009). " La recherche française en sciences humaines et sociales sur les technologies en éducation ". Revue française de pédagogie, n० 169, p. 53-66.

ALTER N. (2000). L'innovation ordinaire. Paris : PUF.

BAREIL C. (2004). La résistance au changement : synthèse et critique des écrits. Cahier no 04-10. Montréal : Centre d'études en transformation des organisations. En ligne : http://web.hec.ca/sites/ceto/fichiers/04_10.pdf.

BARON G.-L. \& BRUILLARD É. (1996). L'informatique et ses usagers dans l'éducation. Paris : PUF.

BARON G.-L. \& BRUILLARD É. (2004). «Quelques réflexions autour des phénomènes de scolarisation des technologies ". In L.-O. Pochon \& A. Maréchal, Entre technique et pédagogie. La création de contenus multimédia pour l'enseignement et la formation. Neuchâtel : IRDP, p. 154-161. En ligne : http://www.stef.ens-cachan.fr/annur/bruillard/GLB_EB_ post_Neuchatel.pdf. 
BILLOUARD D. (2011). Les environnements numériques de travail : proposition d'une démarche d'intégration. Thèse, sciences de l'information et de la communication, université Jean-Moulin-Lyon 3.

BOULLÉ M. (2010). Sécurisation des accès aux espaces numériques de travail. Document ENEIDE (Espace numérique éducatif interactif de demain), STEF. Cachan : ENS Cachan. En ligne : http://www.stef.ens-cachan.fr/ent/eneide_rapport_securisation_acces_boulle_ fev_2010.pdf.

BOURDON I. \& OLLET-HAUDEBERT S. (2009). "Towards an understanding of knowledge management systems - UTAUT Revisited ". Communication présentée au colloque AMCIS 2009, contribution $n^{\circ}$ 445. En ligne : http://aisel.aisnet.org/amcis2009/445.

BRANGIER E., DUFRESNE A. \& HAMMES-ADELÉ S. (2009). « Approche symbiotique de la relation humain-technologie. Perspectives pour l'ergonomie informatique ». Le travail humain, vol. 72, no 4, p. 333-353.

BRUILLARD É. (1997). «L'ordinateur à l'école : de l'outil à l'instrument ». In L.-O. Pochon \& A. Blanchet (dir.), L'ordinateur à l'école : de l'introduction à l'intégration. Neuchâtel : IRDP, p. 99-118.

BRUILLARD É. (2011). «Discours généraux sur les TIC en éducation : beaucoup de slogans peu étayés, en quête de débats ». Epinet, $n^{\circ}$ 138. En ligne : http://www.epi.asso.fr/revue/ articles/a1110e.htm.

BRUILLARD É. \& HOURBETTE D. (2008). « Environnements numériques de travail : un modèle bureaucratique à modifier ? »Argos, n 44, p. 29-34.

CACHEUX C. (2009). "Analyse des usages des espaces numériques de travail dans l'enseignement secondaire. Usages prescrits: adhésion ou résistance des usagers? 》ISDM, no 37. En ligne : http://isdm.univ-tIn.fr/articles/num_archives.htm\#isdm37.

CAISSE DES DÉPÔTS ET CONSIGNATIONS \& CAP DIGITAL (2010). Environnements numériques de travail (ENT) / virtual learning environments (VLE). Marché international de l'eéducation. État des lieux et diagnostic. Rapport dirigé par PM Conseil \& Education Impact. En ligne : http://projets-ent.com/wp-content/uploads/2010/10/CDC_RapportIn ternational_VLE_Juin2010.pdf.

CAISSE DES DÉPÔTS ET CONSIGNATIONS, MINISTÈRE DE L'ÉDUCATION NATIONALE \& EUROPEAN SCHOOLNET (2010). Les plates formes virtuelles d'apprentissage en Europe: que nous apprennent les expériences du Danemark, du Royaume-Uni et de l'Espagne ? Panorama comparatif. En ligne : http://projets-ent.com/wp-content/ uploads/2010/11/Benchmark_european-apprentissage.pdf.

CAISSE DES DÉPÔTS ET CONSIGNATIONS, MINISTÈRE DE L'ÉDUCATION NATIONALE \& SAGATEL (2010). Étude portant sur l'évaluation des besoins en débit des établissements du secondaire équipés d'un ENT. Résumé de l'étude. En ligne : http://www. valoffre.caissedesdepots.fr/IMG/pdf/ENT_CDC_Synthese_etude_10p_v2.pdf.

CARDON D. (2006). « La trajectoire des innovations ascendantes : inventivité, coproduction et collectifs sur Internet ». Communication présentée au colloque «Innovations, usages, réseaux », Montpellier, 17 et 18 novembre 2006. En ligne : http://hal.archives-ouvertes.fr/ docs/00/13/49/04/PDF/Cardon_IUR2006.pdf.

CARON B., CARRON T., CHABERT G. et al. (2004). "L'espace numérique de travail du "cartable électronique®" ». In F. Peccoud \& C. Frasson (dir.), TICE [Technologies de l'information et de la connaissance dans l'enseignement supérieur et l'industrie] 2004. Actes du colloque TICE 2004, Université de technologie de Compiègne, les 21, 22 et 23 octobre 2004. Compiègne : Université de technologie de Compiègne, p. 415-423.

CERISIER J.-F. (dir.) (2005). Environnements numériques de travail : des usages aux analyses d'usages. Paris : CNDP. En ligne : http://rhrt.edel.univ-poitiers.fr/sommaire. php?id=570.

CHAPTAL A. (2005). « Le mammouth et la vache à lait. Réflexions sur les relations entre l'État et les collectivités territoriales en matière de TICE dans l'enseignement scolaire ». Dossiers de l'ingénierie éducative, no 53, p. 78-82.

CHAPTAL A. (2007a). «Paradoxes des usages des TICE : réflexions croisées sur les usages en classe par les enseignants en France, aux États-Unis et au Royaume-Uni ». Dossiers de l'ingénierie éducative, hors série, p. 73-92.

CHAPTAL A. (2007b). "Usages prescrits ou annoncés, usages observés ». Document numérique, vol. $10, \mathrm{n}^{\circ} 3$, p. 81-106. En ligne : www.cairn.info/revue-document-numerique2007-3-page-81.htm.

CHAPTAL A. (2008). TICE: Rule Britannia? Rapport d'étude réalisée pour Cap Digital. En ligne: http://www.pfast.fr/?Rule-Britannia. 
CHEVALIER Y. (2008). Système d'information et gouvernance. Technicité et démocratie à l'université. Fernelmont (Belgique) : EME \& InterCommunications.

CHOPPIN A. (2005). "L'édition scolaire française et ses contraintes : une perspective historique ». In É. Bruillard (dir.), Manuels scolaires, regards croisés. Caen : CRDP de BasseNormandie, p. 39-53.

CHRISTENSEN C., HORN M. \& JOHNSON C. (2008). Disrupting class: How disruptive innovation will change the way the world learns. New York: McGraw-Hill.

COLLET G. (2004). "Étude des conditions de réussite de l'opération "cartable électronique $₫ "$ ". ISDM, n० 18. En ligne : http://isdm.univ-tln.fr/articles/num_archives. htm\#isdm18.

COLLET G., ANSELM D., NARVOR B. \& TEREPA C. (2007). « L'opération "cartable numérique" de Grenoble : ambiguïtés du système et développement des usages ». ISDM, n० 29. En ligne: http://isdm.univ-tln.fr/articles/num_archives.htm\#isdm29.

COLLINS A. \& HALVERSON R. (2009). Rethinking education in the age of technology: The digital revolution and schooling in America. New York: Teachers College Press.

COUTELLIER F., RICHARD G., LE LOUP S. \& BOISSIÈRE J. (2010). Pour une évaluation partagée des usages des espaces numériques de travail. Réalisation d'un outil de mesure d'audience. Paris : Caisse des dépôts et consignations. En ligne : http://projets-ent.com/ wp-content/uploads/2010/11/Guide-ENT_Suivi-partag\%C3\%A9-des-usages.pdf.

CROS F. (1997). « L'innovation en éducation et en formation ». Revue française de pédagogie, no 118, p. 127-156.

CUBAN L. (1986). Teachers and machines. The classroom use of technology since 1920. New York : Teachers College Press.

DAGUET H. \& VOULGRE E. (2011). « Discours et pratiques autour des environnements numériques de travail. Utopie ou réalité ? » In M. Bétrancourt, C. Depover, V. Luengo et al. (dir.), À la recherche de convergence entre les acteurs des environnements informatisés d'apprentissage humains, EIAH 2011. Mons : Éd. de l'Université de Mons, p. 231241.

DAVIS F. (1985). A technology acceptance model for empirically testing new end-user information systems: Theory and results. Thèse, management, Massachusetts Institute of Technology, Sloan School of Management. En ligne : http://dspace.mit.edu/han dle/1721.1/15192.

DAVIS F. (1993). « User acceptance of information technology: System characteristics, user perception and behavioural impact ». International Journal Man-machine Studies, n 38, p. 475-487.

DIEUZEIDE H. (1982). «Marchands et prophètes en technologie de l'éducation ». In Actes du colloque «Les formes médiatisées de la communication éducative ». Saint-Cloud : École normale supérieure de Saint-Cloud, p. 78-82. En ligne: http://halshs.archives-ouvertes. $\mathrm{fr} /$ docs/00/02/92/61/PDF/dieuzeide82.pdf.

ECKHARDT A., LAUMER S. \& WEITZEL T. (2009). «Who influences whom? Analyzing workplace referents' social influence on IT adoption and non-adoption ". Journal of Information Technology, vol. 24, no 1, p. 11-24.

FERRARIS C., LEJEUNE A., VIGNOLLET L. \& DAVID J.-P. (2005). « Modélisation de scénarios pédagogiques collaboratifs ». In P. Tchounikine, M. Joab \& L. Trouche (dir.), EIAH 2005 (Environnements informatiques pour l'apprentissage humain). Actes de la conférence EIAH 2005, Montpellier 25, 26 et 27 mai. Lyon : INRP.

FÉVRIER F., JAMET É. \& ROUXEL G. (2008). "Quel outil d'évaluation de l'acceptabilité des nouvelles technologies pour des études francophones ? " In IHM 2008, Metz. 20 ans d'interaction homme-machine francophone: de l'interaction à la fusion entre l'humain et la technologie. New York : ACM Press, p. 199-204.

FÉVRIER F., GAUDUCHEAU N., JAMET É., ROUXEL G. \& SALEMBIER P. (2011). « La prise en compte des affects dans le domaine des interactions homme-machine : quels modèles, quelles méthodes, quels bénéfices ? " Le travail humain, vol. 74, n 2, p. 183-201.

FORD J., FORD L. \& D'AMELIO A. (2008). "Resistance to change: The rest of the story ». Academy of Management Review, vol. 33, n² 2, p. 362-377.

GENEVOIS S. \& POYET F. (2009a). «Espaces numériques de travail (ENT) et "école étendue". Vers un nouvel espace-temps scolaire? " Distances et savoirs, vol. 8, no 4, p. 565-583.

GENEVOIS S. \& POYET F. (2009b). Les usages pédagogiques des ENT d'lsère et d'Auvergne. Rapport d'étude. Lyon : INRP. 
GINIOUX P. \& NARCY M. (2002). « Le cartable électronique ». Médialog, n 43. En ligne : http://medialog.ac-creteil.fr/ARCHIVE43/cartable43.pdf.

GROS A.-M. (2001). «Un bureau virtuel pour l'enseignant ». Médialog, no 41. En ligne : http://medialog.ac-creteil.fr/ARCHIVE41/bureauvi.pdf.

HÉNOCQ S. (2008). Les discours des enseignants innovateurs en sciences de la vie et de la Terre sur les activités qu'ils mènent avec les espaces numériques de travail. Mémoire de master 2, didactique des sciences et des technologies, École normale supérieure de Cachan.

HUMBERT P. (2010). «Pilotage de la conception d'outils numériques ». Les cahiers du numérique, vol. 6 , no 4 , p. 49-75.

IDATE (2003). Étude internationale sur les environnements numériques de travail. Rapport de synthèse. En ligne : http://www.abhatoo.net.ma/index.php/fre/Maalama-Textuelle/ Sciences-de-I\%27information/Technologies-de-I\%27information-et-de-la-communica tion-TIC/Applications-sectorielles-des-TIC/E-learning/Etude-internationale-sur-lesenvironnements-num\%C3\%A9riques-de-travail-Rapport-de-synth\%C3\%A8se.

IJAZ S. \& VITALIS A. (2011). "Resistance to organizational change: Putting the Jigsaw together ». International review of business research papers, vol. 7, n० 3, p. 112-121. En ligne : http://www.bizresearchpapers.com/8.\%20Saima-FINAL.pdf.

JAURÉGUIBERRY F. (2010). «Les théories sur le non-usage des technologies de communication ". In Actes des travaux du comité de recherche "Sociologie de la communication ». Colloque AISLF, Namur, 19 au 20 mai 2010. Namur (Belgique) : Facultés universitaires Notre-Dame de la Paix, p. 22-30. En ligne : http://w3.aislf.univ-tlse2.fr/gtsc/ DOCS_SOCIO/2010/Actes_AISLF_CR33_Namur_2010.pdf\#page=22.

KAPLAN D. (dir.) (2002). Les cartables électroniques. Rapport du groupe de travail de la FING. Paris : Fondation pour l'Internet nouvelle génération. En ligne : http://www.artesi.artesiidf.com/public/anv/document.srv?id=6001.

KAPLAN D. \& POUTS-LAJUS S. (2004a). " Les ENT dans le monde, des modèles d'une grande diversité ". Dossiers de l'ingénierie éducative, n० 46.

KAPLAN D. \& POUTS-LAJUS S. (dir.) (2004b). Du cartable électronique aux espaces numériques de travail. Paris : La Documentation française.

KELLNER C., MASSOU L. \& MORELLI P. (dir.) (2010). «(Re)penser le non-usage des TIC ». Questions de communication, no 18, p. 7-20.

KHANEBOUBI M. (2009). « Description de quelques caractéristiques communes aux opérations de dotations massives en ordinateurs portables en France ». STICEF, vol. 16. En ligne : http://sticef.univ-lemans.fr/num/vol2009/06r-khaneboubi/sticef_2009_khane boubi_06.htm.

LAPOINTE L. \& RIVARD S. (2005). «A multilevel model of resistance to information technology implementation ». MIS Quarterly, vol. 29, n 3, p. 461-491.

LAULAN A.-M. (1985). La résistance aux systèmes d'information. Paris : Retz.

MARCHEWKA J., LIU C. \& KOSTIWA K. (2007). " An application of the UTAUT model for understanding student perceptions using course management software ». Communications of the IIMA, vol. 7, n० 2, p. 93-104.

MARQUET P. \& DINET J. (2003). « Un cartable numérique au lycée : élément de sa genèse instrumentale chez les enseignants et les élèves ». In C. Desmoulins, P. Marquet \& D. Bouhineau (dir.), EIAH 2003 (Environnements informatiques pour l'apprentissage humain). Actes de la conférence EIAH 2003, Strasbourg, 15, 16 et 17 avril. Lyon : INRP, p. 307-318. En ligne : http://hal.archives-ouvertes.fr/docs/00/00/16/67/PDF/n029-48.pdf

MARQUET P. \& DINET J. (2004). « Les premiers usages d'un cartable numérique par les membres de la communauté scolaire : un exemple en lycée ". Revue française de pédagogie, no 146, p. 79-91.

MARTEL C. \& VIGNOLLET L. (2001). « Educational web portal on personalized and collaborative services ». In Actes de la conférence internationale IEEE «Advanced learning technologies ». En ligne: http://dx.doi.org/10.1109/ICALT.2001.943932.

MARTEL C., FERRARIS C., CARON B. et al. (2004). " A model for CSCL allowing tailorability: Implementation in the electronic schoolbag groupware ". In CRIWG 2004: International workshop on groupware no 10, San Carlos, Costa Rica. Berlin : Springer, p. 322-337. En ligne : http://www.syscom.univ-savoie.fr/publi/Martel2004a.pdf.

MEISSONIER R., HOUZE E. \& BELBALY N. (2007). « Résistance aux projets d'implantation de technologies de l'information : le cas d'une PME du secteur des hautes technologies ». Gestion, vol. 32, no 1, p. 20-27. 
MINISTÈRE DE L'ÉDUCATION NATIONALE (2010). Plan de développement des usages du numérique à l'école. Dossier de presse. Paris : Ministère de l'Éducation nationale. En ligne : http://www.education.gouv.fr/cid54064/plan-de-developpement-des-usages-dunumerique-a-l-ecole.html.

MISSONIER S. (2008). Comprendre pour aider. Analyse réticulaire de projets de mise en œuvre d'une technologie de l'information: le cas des espaces numériques de travail. Thèse, sciences de gestion, université de Nice. En ligne : http://tel.archives-ouvertes.fr/ tel-00368354_v1/.

MCEGLIN P. (2010). Les industries éducatives. Paris : PUF.

PACURAR E., ABBAS N. \& MOLTINI-ZENDER S. (2011). «Quels usages effectifs de I'ENT par les enseignants ? "In M. Bétrancourt, C. Depover, V. Luengo et al. (dir.), À la recherche de convergence entre les acteurs des environnements informatisés d'apprentissage humains, EIAH 2011. Mons : Éd. de I'Université de Mons, p. 243-257.

POUTS-LAJUS S. (2005). "Classification des projets ENT et comparaisons internationales ». In Actes des 5 et 6 e rencontres réseaux humains, réseaux technologiques. Poitiers et La Rochelle, 16 et 17 mai 2003, 25 et 26 juin 2004. Paris : CNDP, p. 25-35. En ligne : http://rhrt.edel.univ-poitiers.fr/document.php?id=735.

POYET F. \& BACONNIER B. (2006). « Les environnements numériques de travail en milieu scolaire ". Lettre d'information de la veille scientifique et technologique, n० 21. En ligne : http://ife.ens-lyon.fr/vst/DA/detailsDossier.php?parent=accueil\&dossier=21\&lang=fr.

POYET F. \& GENEVOIS S. (2007). « Impact des objets métaphoriques sur l'utilisation d'un ENT au collège ». Communication présentée au congrès international AREF 2007 (Actualité de la recherche en éducation et en formation), Strasbourg. En ligne : http://www. congresintaref.org/Acte269.html.

PUIMATTO G. (2004). « Un historique et un panorama ». Dossiers de l'ingénierie éducative, n० 46.

PUIMATTO G. (2005). « Réseaux numériques éducatifs ». Distances et savoirs, vol. 3, n 3, p. 283-309.

PUIMATTO G. (2006). Les réseaux numériques éducatifs régulateurs, acteurs et vecteurs de l'évolution des pratiques et de l'organisation des établissements et de l'institution scolaires. Thèse, sciences de l'information et de la communication, université Paris 13 . En ligne : http://archive-edutice.ccsd.cnrs.fr/docs/00/09/55/87/PDF/These_GP_release_4. pdf.

PUIMATTO G. (2008). « Supérieur et scolaire, même combat ». Argos, no 44, p. 35-41.

QUENTIN I. (2011). Analyse de documents de prospective en éducation. Rapport interne. Cachan : ENS Cachan. En ligne : http://www.stef.ens-cachan.fr/docs/quentin_prea2k30_ rapport_2011.pdf.

RABARDEL P. (1995). Les hommes et les technologies. Approche cognitive des instruments contemporains. Paris : Armand Colin.

RINAUDO J.-L. \& POYET F. (2009). Les environnements numériques en milieu scolaire. Quels usages et quelles pratiques? Lyon: INRP.

ROGERS E. (1995). Diffusion of innovations [1962]. New York : Free Press.

ROMBY A. (2001). « Les technologies, des vecteurs de changement ? Le cas des technologies de l'information et de la communication dans les lycées de Picardie: le projet Éducapôle ». Éducation et sociétés, no 8, p. 143-157.

SALAÜN J.-M. (2008). «Web, texte, conversation et redocumentarisation ». In Actes des ge journées internationales d'analyse statistique des données textuelles, Lyon, 12-14 mars 2008. Lyon : Presses universitaires de Lyon, p. 27-30. En ligne : http://hdl.handle. net/1866/2226.

SCHNEEWELE M., CHERQUI-HOUOT I., NOWAKOWSKI S., TRESTINI M., ZAPATA A. \& PACURAR E. (2010). «Implantation d'un ENT dans l'enseignement secondaire, analyse et modélisation des usages : le cas lorrain ». Communication au $7^{e}$ colloque des technologies de l'information et de la communication pour l'enseignement, Nancy. En ligne : http://hal.archives-ouvertes.fr/hal-0059215.

TOULOUSE E. (2002). « Le cartable électronique de Savoie ». Dossier de l'ingénierie éducative, n० 41.

ULLMANN C. (2007). «Les régions françaises dans le millefeuille institutionnel des politiques de développement numérique ». Networks and Communication Studies, vol. 21, n० 1-2, p. 113-136. 
VENKATESH V. \& ZHANG X. (2010). « Unified theory of acceptance and use of technology: US vs. China ». Journal of Global Information Technology Management, vol. 13, no 1, p. 5-27.

VENKATESH V., MORRIS M., DAVIS G. \& DAVIS F. (2003). « User acceptance of information technology: Toward a unified view ». MIS Quarterly, vol. 27, n० 3, p. 425-478.

VIALLE B. (2001). L'expérimentation assistée par ordinateur dans l'enseignement des sciences de la vie au lycée. Aide et obstacle à la rénovation de l'approche expérimentale. Thèse, sciences de l'éducation, université de la Réunion.

VON HIPPEL E. (2005). Democratizing innovation. Cambridge : MIT Press.

VOULGRE E. (2010). "Espace numérique de travail en collège : étude sur la formation des enseignants ». Distances et savoirs, vol. 8, no 4, p. 585-600.

VOULGRE E. (2011). Une approche systémique des TICE dans le système scolaire français : entre finalités prescrites, ressources et usages par les enseignants. Thèse, sciences de l'éducation, université de Rouen. 
